Universidade de Brasília

Instituto de Física

Dissertação de mestrado

\title{
Solução analítica para o modelo RSOS
}

\author{
Waldenor Ramone Juvito Gomes
}

Brasília, Julho de 2016 


\title{
Solução analítica para o modelo RSOS
}

\author{
Waldenor Ramone Juvito Gomes
}

Orientador:

Prof. Dr. Fernando Albuquerque de Oliveira

Co-orientador:

Prof. Dr. Ismael Victor de Lucena Costa

Brasília, Julho de 2016 


\section{Agradecimentos}

"Se cheguei mais longe foi por estar de pé sobre ombros de gigantes" ${ }^{1}$. Esta citação define bem toda minha jornada e é para estes gigantes que eu agradeço.

Primeiramente ao apoio total dos meus pais, Maria Francelina Juvito Gomes (infelizmente não está perto de mim) e Damião Gomes Cazé, que me ensinaram os valores da ética, da cidadania e do estudo. Agradeço as oportunidades que eles me deram. Também ao apoio da minha noiva, futura esposa e eterna namorada, Kamila Franco Paiva que está comigo desde 21/11/2011 nos bons e maus momentos e me incentivando a ir mais longe. Esta conquista, que não é mais somente minha, mas é nossa primeira de muitas conquistas que teremos na nossa vida juntos.

Agradeço ao meu Orientador e meu Co-orientador, professor Dr. Fernando Albuquerque de Oliveira e professor Dr. Ismael Victor de Lucena Costa, respectivamente. Ambos me acolheram nesse meu início de caminhada na vida acadêmica e com muito empenho e dedicação me ensinaram e espero corresponder a confiança deles. Fico grato a todos os professores do IF-UnB que fizeram parte da minha graduação.

Aos meus amigos: Lucas Holanda, Rodrigo, Moises, Lydiane, Deborah, Artur, Solano, Mirian, Evandro, Carol, que me ajudaram bastante neste meu trabalho e me propiciaram momentos de descontração fundamental para qualquer pessoa. Ao Rodrigo e Mirian um muito obrigado pela ajuda na parte de computação que foi fundamental para a conclusão desta dissertação. Agradeço a Antonio Beserra pela grande ajuda em corrigir meus erros de Língua Portuguesa. E a todos meus amigos, cujos agradecimentos não caberiam nas páginas deste livro.

Também ao meu professor de Física do Ensino Médio Humberto Nei, principal incentivador para que eu seguisse na carreira de Física e me auxiliando durante meus estágios obrigatórios.

Agradeço à CAPES e a UnB por todo apoio financeiro.

\footnotetext{
${ }^{1}$ Isaac Newton
} 


\section{Resumo}

Nesta dissertação iremos analisar a dinâmica do crescimento para modelos de autômatos celulares. Uma vez que a evolução cria uma superfície de fractal, mostraremos que a variação da rugosidade pode ser apresentada em uma expressão simples que combina duas componentes: a distribuição de probabilidade das configurações da superfície e as regras que define um autômato celular. A partir destas considerações investigaremos uma das variações do modelo Restricted Solid-On-Solid (RSOS) em 1+1 dimensão e as propriedades da rugosidade, assim como as propriedades gerais que a mesma deve satisfazer.

Palavras-Chaves: Modelo de crescimento, expoentes críticos, dinâmica de crescimento, equação KPZ, autômato celular. 


\section{Abstract}

This dissertation will examine the growth dynamics for cellular automata models. Since the evolution creates a fractal surface, we show that the variation of roughness can be presented in simple expression that combines two components: the probability distribution of the surface configurations and the rules that define a cellular automata. From these considerations we will investigate one of the variations of the Restricted Solid-On-Solid (RSOS) model in 1+1 dimension and properties of the roughness, as well as the general properties that it must satisfy.

Key words: Growth model, critical exponents, growth dynamics, KPZ equation, cellular automata. 


\section{Sumário}

Sumário vi

Lista de Figuras $\quad$ ix

1 Introdução 1

2 Conceitos Básicos 3

2.1 Altura média e Rugosidade . . . . . . . . . . . . . . . . . 3

2.1.1 Altura média . . . . . . . . . . . . . . 5

2.1.2 Rugosidade .................... . . 5

2.2 Leis de escalas e expoentes críticos . . . . . . . . . . . . 6

$\begin{array}{lll}3 & \text { Dinâmica de crescimento } & 10\end{array}$

3.1 Príncipios de simetria . . . . . . . . . . . . . . . . . . . 12

3.1 .1 Invariância sob translação temporal . . . . . . . . . . . . . . . 12

3.1.2 Invariância na translação ao longo da direção do crescimento . 12

3.1.3 Invariância na translação na direção perpendicular à direção do crescimento . . . . . . . . . . . . . . . 13

3.1.4 Rotação e inversão na direção do crescimento . . . . . . . . . 13

3.1.5 Simetria Up/Down para a altura . . . . . . . . . . . 13

3.2 A equação de Edwards-Wilkinson . . . . . . . . . . . . . . . . 13

3.3 A equação de Kardar-Parisi-Zhang . . . . . . . . . . . . . . . . . . . 15

3.3 .1 Construção da equação KPZ . . . . . . . . . . . . . . . . . . 15 
3.4 Propriedades da equação KPZ . . . . . . . . . . . . . . . . . . 17

3.4.1 Invariância de Galileu . . . . . . . . . . . . . . . . . . 17

3.5 Problemas atuais da Física de crescimento . . . . . . . . . . . . . . . 19

3.5.1 Validade da invariância de Galileu . . . . . . . . . . . . . . 19

3.5.2 Dimensão crítica superior . . . . . . . . . . . . . . 20

3.5.3 Autômatos celulares e KPZ . . . . . . . . . . . . . . . 20

4 Evolução da rugosidade em modelos de autômatos celulares $\quad 21$

4.1 Modelo RSOS . . . . . . . . . . . . . . . . . . . . . . 22

4.1 .1 Simulações do Modelo RSOS . . . . . . . . . . . . . . . . . 23

4.2 Descrição da teoria para MAC . . . . . . . . . . . . . . . . 25

4.2.1 Obtenção da FDE . . . . . . . . . . . . . . . . . 26

4.2.2 Normalização da probabilidade . . . . . . . . . . . . . . 28

4.2.3 Considerações gerais sobre a teoria . . . . . . . . . . . . . 29

4.3 Aplicação da teoria para o modelo RSOS . . . . . . . . . . . . . . . . 30

4.3 .1 Caso a . . . . . . . . . . . . . . . . 31

4.3 .2 Caso b . . . . . . . . . . . . . . . . . . 32

4.4 Obtenção do expoente de rugosidade . . . . . . . . . . . . . . . . 33

4.4.1 O destino de uma teoria . . . . . . . . . . . . . 33

5 Conclusão $\quad 35$

$\begin{array}{ll}\text { A Incremento de rugosidade } & 37\end{array}$

A.1 Desenvolvimento do incremento da rugosidade . . . . . . . . . . . 37

B Função de distribuição de equiprobabilidade (FDE) 39

B.1 Obtenção da função $f\left(\xi_{1}, \xi_{2}, \xi_{3}, \omega\right) \ldots$. . . . . . . . . . . . 39

C Função Gama 42

C.1 Função Gama . . . . . . . . . . . . . . . . . . . . . . . . . . . . . . 42

C.1.1 Fórmula de Stirling . . . . . . . . . . . . . . . . . 43 
C.1.2 Função Beta . . . . . . . . . . . . . . . . . . . . 44

Referências Bibliográficas 


\section{Lista de Figuras}

2.1 Esquema geral de um substrato . . . . . . . . . . . . . . . . . . 4

2.2 Crescimento de um sistema obtido por simulação [1] e [2] . . . . . . . 7

2.3 Evolução temporal da largura da superfície para diferentes valores de L. [2] . . . . . . . . . . . . . . . . . . . . . . . . . . . 8

2.4 Variação da rugosidade com o tempo para três diferentes valores de L. Fonte: Barabási \& Stanley. . . . . . . . . . . . . . . . . . . . 8

3.1 Formação de superfíce entre dois meios. [3] . . . . . . . . . . . . . . 10

3.2 A origem do termo não linear na equação KPZ. . . . . . . . . . . . . 16

4.1 Configurações para o modelo RSOS. . . . . . . . . . . . . . . . . . 22

4.2 Evolução temporal da rugosidade. . . . . . . . . . . . . . . . 23

4.3 Evolução temporal da rugosidade (normalizado). O gráfico é uma gentiliza do Dr. Evandro A. Rodrigues . . . . . . . . . . . . . . . . . 24

$4.4 \omega_{\text {sat }}$ em função do comprimento $L \ldots \ldots$. . . . . . . . . . . . . . . 25

4.5 Normalização da probabilidade em função de $L$. . . . . . . . . . . . 29 


\section{Capítulo 1}

\section{Introdução}

Este trabalho é dedicado ao estudo do crescimento, em particular fixaremos nossa atenção na rugosidade que aparece na interface entre dois meios durante o processo de crescimento. A formação de interface entre superfícies pode ser observada facilmente no nosso cotidiano: formação de líquens em caule de árvores e rochas, a formação do mofo nas paredes de uma casa, ao viajar perto de regiões montanhosas observamos a erosão no solo devido a ação da chuva[4], em solos de regiões áridas ou semiáridas expostas ao clima desértico, além dos fenômenos estudados em laboratórios, por exemplo em ciências dos materiais a interface entre duas superfícies apresenta uma série de propriedades que necessitam ser bem determinadas. Em biologia temos o estudo de crescimento de colônias de bactérias, a interface entre uma célula e o meio externo etc. Na natureza os exemplos são inesgotáveis. O objetivo deste trabalho será fazer uma descrição geral desses fenômenos, descrever cada regime do processo de crescimento, e os expoentes críticos $\alpha, \beta$ e $z$, associados com ele, bem como as relações entre esses expoentes, e "o estado da arte" atual na área.

No capítulo 02 apresentaremos os conceitos necessários para análise de um modelo de crescimento, i.e., tal como para descrever a transição líquido-gás usamos as grandezas calor específico $\left(C_{V}\right)$, densidade $(\rho)$ e compressibilidade isotérmica $\left(\kappa_{T}\right)$ onde proxímo ao ponto crítico $T \sim T_{c}$ ( $T_{c}$ é a temperatura crítica), 
essas grandezas comportam-se como leis de potência da forma $C_{V} \sim\left|T_{c}-T\right|^{-\alpha}$, $\rho_{c}-\rho \sim\left|T_{c}-T\right|^{\beta}$ e $\kappa_{T} \sim\left|T_{c}-T\right|^{-\gamma}$, aqui $\alpha, \beta$ e $\gamma$ são chamados expoentes críticos. Na Física de crescimento as grandezas importantes são a altura média $(\bar{h})$, a rugosidade $(\omega)$ e seus expoentes $\alpha, \beta$ e $z$ que serão definidos. E por fim é apresentado a relação de escala de Family-Vicsek onde obtemos a relação entre os expoentes críticos.

No capítulo 03 iniciamos a discurssão sobre a dinâmica de crescimento, onde apresentamos os conceitos de simetria que delimitam as possibilidades de uma equação de crescimento que descreva a evolução de uma superfície. Deste modo as possibilidades mais simples são: a equação linear de Edwards-Wilkinson (EW) e a não-linear de Kardar-Parisi-Zhang (KPZ). Encerramos o capítulo citando alguns problemas atuais no campo da Física de crescimento.

No capítulo 04 apresentamos o tema central do trabalho: a Evolução da rugosidade aplicada para autômato celular. Inicialmente descrevemos o modelo RSOS (Restricted Solid-On-Solid) [5], seu algoritmo e as configurações possíveis. Em seguida tratamos das simulações feitas para este modelo. Para uma descrição teórica da rugosidade utilizamos um método utilizado por Alves e colaboradores [1, 6] onde eles obtêm a rugosidade para o modelo de corrosão [7]. Então aplicaremos a teoria ao modelo RSOS para estudar a evolução da rugosidade do sistema e obter o valor do expoente de rugosidade $\alpha$. Neste capítulo mostramos também alguns resultados gerais que devem vir da teoria.

No capítulo 05 concluímos nosso trabalho resumindo os resultados obtidos descritos nessa dissertação. 


\section{Capítulo 2}

\section{Conceitos Básicos}

Para a explicação dos modelos de crescimento é necessário conhecer alguns parâmetros que caracterizam estes modelos. Serão tratados neste capítulo os conceitos básicos para o estudo de modelos de crescimento, seja o RSOS, o modelo de corrosão, o modelo de deposição aleatória [8] ou deposição aleatória com Relaxação Superficial [9], além de muitos outros em estudo [2].

Dizemos que dois modelos pertencem a mesma classe de universalidade quando eles têm os mesmos expoentes críticos. Deste modo para que possamos classificar cada modelo dentro de uma classe de universalidade é preciso conhecer os seus expoentes críticos - $\alpha$ expoente de rugosidade, $\beta$ o expoente de crescimento e $z$ o expoente dinâmico - bem como a relação de dependência que existe entre estes expoentes.

\subsection{Altura média e Rugosidade}

Os processos de crescimento começam com uma definição do modelo empregado para sua dinâmica, sendo o mecânismo mais comum o da deposição. Podemos representar um modelo de deposição de partículas como exibido na figura (2.1): 


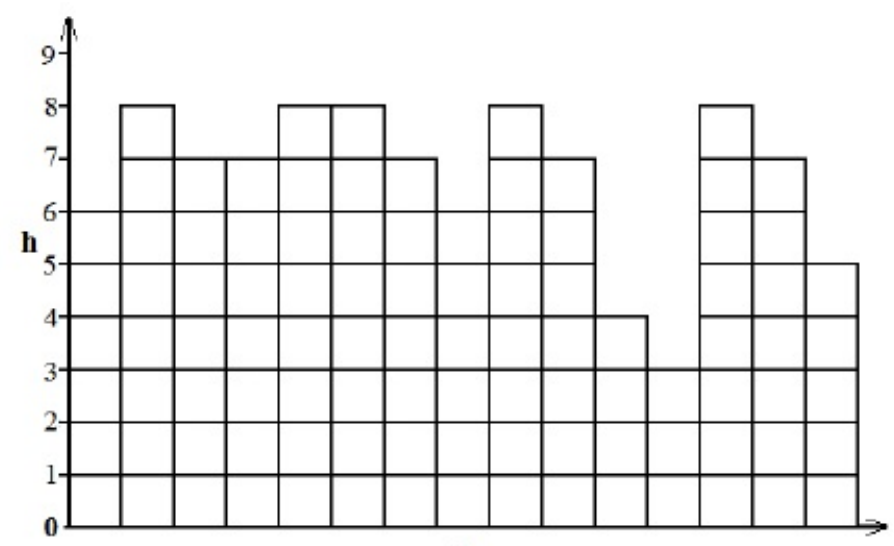

$\mathbf{L}$

Figura 2.1: Esquema geral de um substrato

Considerando $L$ o tamanho do substrato, a figura descrita acima é representado por coordenadas cartesianas, em que o tamanho do substrato correspondente ao eixo das abscissas é dado sob a forma $0 \leq i \leq L$, onde $i$ representa o local onde as partículas devem ser depositadas. Essa altura é representada por $h(i, t)$ ou $h_{i}(t)$ que corresponde o valor da altura do local $i$ num dado instante $t$ com relação a um referencial fixo.

Neste trabalho faremos uso de modelos de crescimento discretos. Para um modelo discreto, o mecanismo de crescimento é descrito por um algoritmo ${ }^{2}$ (Autômato Celular) no intuito de tentar reproduzir, satisfatoriamente, vários aspectos de algum tipo de crescimento observado experimentalmente.

Para descrever um modelo de crescimento quantitativamente iremos introduzir duas grandezas: a altura média e a rugosidade.

\footnotetext{
${ }^{2}$ Mecanismos encarregados tanto da difusão quanto da fixação das partículas sob a superfície. Por exemplo, para Deposição Aleatória primeiro em um tempo $t$ sorteamos um sítio $i$ com altura $h_{i}(t)$, depois em um tempo subsequente $t+1$ fazemos $h_{i}(t)+1$
} 


\subsubsection{Altura média}

A altura média de uma superfície, denotada por $\bar{h}$, é definida por

$$
\bar{h}(t) \equiv \frac{1}{L} \sum_{i=1}^{L} h_{i}(t)
$$

onde $h_{i}(t)$ é a altura da coluna $i$ no tempo $t$. Por exemplo, caso a taxa de deposição (número de partículas chegando em um sítio $i$ ) seja constante pela (2.1) calculamos que a altura média cresce linearmente com o tempo.

\subsubsection{Rugosidade}

A largura da interface que caracteriza a rugosidade de uma interface, é definida pelo desvio quadrático médio da altura,

$$
\sigma_{h}=\omega(L, t) \equiv \sqrt{\frac{1}{L} \sum_{i=1}^{L} \xi_{i}^{2}(t)}
$$

onde

$$
\xi_{i}(t)=h_{i}(t)-\bar{h}(t)
$$

é a flutuação da altura.

Monitoramos o processo de rugosidade quantitativamente quando medimos a largura da superfície em função do tempo. Por definição, o crescimento começa a partir de uma linha horizontal; a superfície no tempo zero é simplesmente uma linha reta com largura zero, i.e. $\omega(0)=0$. Após as deposições é que a superfície gradualmente começa a apresentar a rugosidade. Observe que esta definição faz sentido físico já que a flutuação de uma grandeza está associada com uma função resposta do sistema. Podemos comparar com um sistema termodinâmico de $N$ 
partículas onde a flutuação da energia

$$
\sigma_{E}^{2}=\left\langle(E-\langle E\rangle)^{2}\right\rangle=k_{B} T^{2} C_{V}
$$

onde $C_{V}$ é o calor específico molar a volume constante e a flutuação do volume

$$
\sigma_{V}^{2}=\left\langle(V-\langle V\rangle)^{2}\right\rangle=T V \kappa_{T}
$$

onde $\kappa_{T}$ é a compressibilidade isotérmica [10].

\subsection{Leis de escalas e expoentes críticos}

Um dos conceitos modernos usados para estudar vários processos de rugosidade é o escalonamento. Escalonamento possui uma surpreendente poder de predição, consiste de simples manipulações que nos permite conectar grandezas e expoentes aparentemente independentes.

A análise das relações de escalonamento nos permite obter os expoentes e definir a classe de universalidade. O conceito de classe de universalidade é um resultado da mecânica estatística moderna, e codifica o fato que há alguns fatores dinâmicos que são universais e determinam os expoentes independentemente do modelo específico. Portanto, diferentes sistemas que aparentamente não possuem nada em comum, mas que pertencem à mesma classe de universalidade, comportamse de forma muito semelhante na vizinhança do ponto crítico.

Na figura (2.2) mostramos a evolução da rugosidade em função do tempo. Partindo inicialmente de uma superfície lisa de modo que $\omega(0)=0$. A linha vertical $t=t_{\text {sat }}$ separa 3 escalas de tempo. Para $t \ll t_{\text {sat }}$ a rugosidade cresce como

$$
\omega(L, t) \sim t^{\beta} \text { para } t \ll t_{\text {sat }} .
$$

O expoente $\beta$ é definido como o expoente de crescimento, que caracteriza as informações a respeito da dinâmica temporal do processo de rugosidade. Pelo gráfico (2.2) observamos que a evolução de $\omega(L, t)$ cessa em um dado instante. Para um 


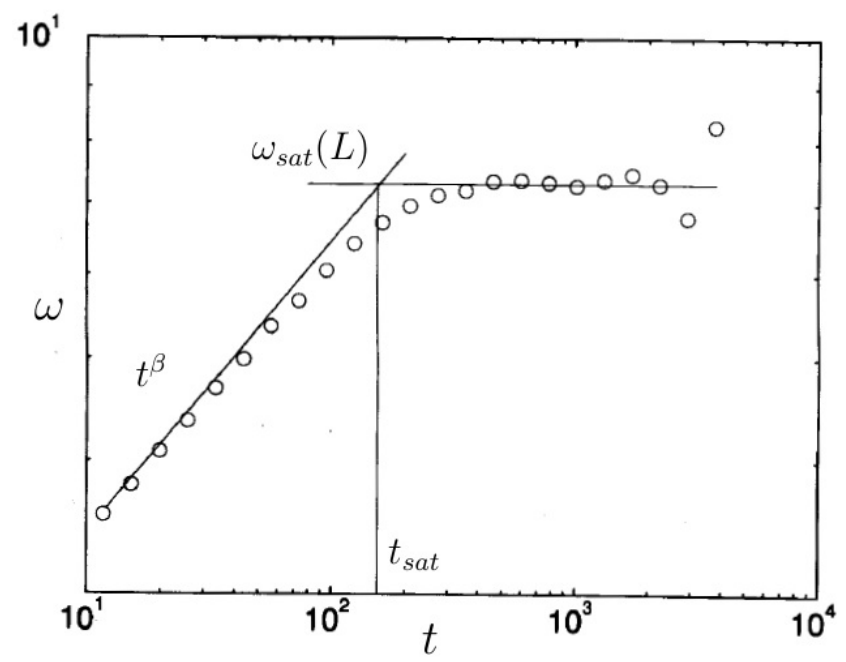

Figura 2.2: Crescimento de um sistema obtido por simulação [1] e [2]

tempo maior que $t_{\text {sat }}$ ocorre o processo de saturação e a rugosidade atinge um valor máximo $\omega_{\text {sat }}$. À medida que aumenta o tamanho $L$ do sistema, a largura de saturação também aumenta como uma lei de potência,

$$
\omega_{\text {sat }}(L) \sim L^{\alpha}
$$

O expoente $\alpha$, chamado de expoente de rugosidade, caracteriza as informações a respeito da rugosidade do sistema quando ocorre a saturação da superfície. O tempo de saturação $t_{\text {sat }}$ também depende do tamanho do sistema e satisfaz uma relação na forma

$$
t_{\text {sat }} \sim L^{z}
$$

onde $z$ é chamado expoente dinâmico do sistema.

Os expoentes $\alpha, \beta$ e $z$ não são independentes, a seguir apresentaremos a relação entre estes expoentes. No gráfico da figura (2.3) - em escala logarítmica - da rugosidade em função do tempo, onde cada curva no gráfico representa um sistema com um certo tamanho L. Podemos colapsar os dados do gráfico (2.3) em uma única curva [11], isto é, faremos um reescalonamento da dependência temporal da 
rugosidade.

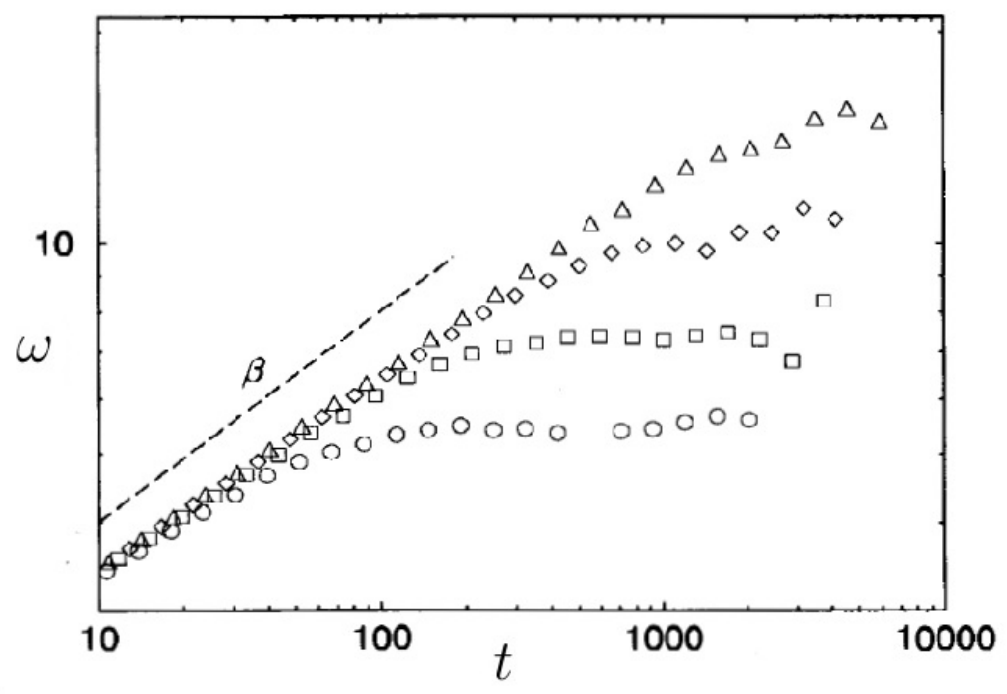

FiguRA 2.3: Evolução temporal da largura da superfície para diferentes valores de L. [2]

Na figura (2.4) representa a evolução temporal da rugosidade para diferentes comprimentos $L$.

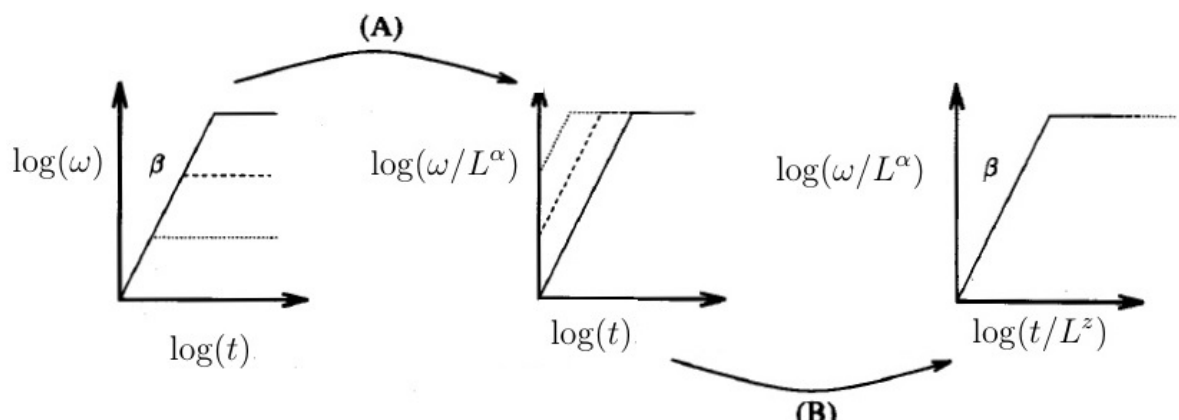

(B)

Figura 2.4: Variação da rugosidade com o tempo para três diferentes valores de $L$.

Fonte: Barabási \& Stanley.

No passo (A) a rugosidade $\omega$ é dividida por $L^{\alpha}$, isto é, pela rugosidade de saturação $\omega_{\text {sat }}$ e plota-se um gráfico, em escala logarítmica, $\omega(L, t) / \omega_{\text {sat }}(L)$ em 
função do tempo, que corresponde a uma mudança vertical das curvas na escala loglog, resultando em curvas que saturam com o mesmo valor, independente do tamanho do sistema. No passo (B) o tempo $t$ é dividido pelo tempo de saturação $t_{s a t} \sim L^{z}$ e novamente plota-se agora um gráfico, em escala logarítmica de $\omega(L, t) / \omega_{\text {sat }}(L)$ em função de $t / t_{\text {sat }}$. Este passo corresponde a uma mudança horizontal da curva que agora satura para um mesmo valor da abscissa, i.e., causando a saturação em um mesmo tempo característico. Todas as curvas colapsam em uma só mostrando o comportamento universal do sistema.

Conclui-se então que $\frac{\omega(L, t)}{\omega_{s a t}(L)}$ é uma função de $\frac{t}{t_{s a t}}$, i.e.,

$$
\frac{\omega(L, t)}{\omega_{\text {sat }}(L)}=f\left(\frac{t}{t_{\text {sat }}}\right)
$$

deste modo tem-se a Relação de Escala de Family-Vicsek onde [2],

$$
f(u)= \begin{cases}u^{\beta}, & u \ll 1 \\ 1, & u \gg 1\end{cases}
$$

Na figura 2.2 se nos aproximarmos do ponto de saturação $\left(t_{\text {sat }}, \omega\left(t_{\text {sat }}\right)\right)$ pela esquerda encontramos, segundo a equação $(2.4)$ que $\omega\left(t_{\text {sat }}\right) \sim t_{\text {sat }}^{\beta}$. Contudo quando aproximamos pelo lado direito ao mesmo ponto temos, segundo $(2.5), \omega\left(t_{\text {sat }}\right) \sim L^{\alpha}$. Assim temos que:

$$
t_{\text {sat }}^{\beta} \sim L^{\alpha}
$$

logo, pela equação (2.6) pode-se concluir que

$$
z=\frac{\alpha}{\beta} .
$$

A equação (2.9) é a lei de escala que conecta os três expoentes e é válida para qualquer processo de crescimento. 


\section{Capítulo 3}

\section{Dinâmica de crescimento}

Nos estudos de modelos de crescimento [12, 13, 7] estamos interessados na posição $h(r, t)$ de uma superfície entre dois meios (3.1), por exemplo, durante um processo de crescimento através da agregação de material de um dos meios para o outro. O termo genérico "crescimento"é usado no sentido de uma evolução de situações físicas tais como a propagação de uma frente de fogo [14, 15, 16], erosão do solo [4], dispersão do fogo em uma floresta, o fluxo de um líquido em um meio poroso ou o crescimento de uma colônia de bactéria.

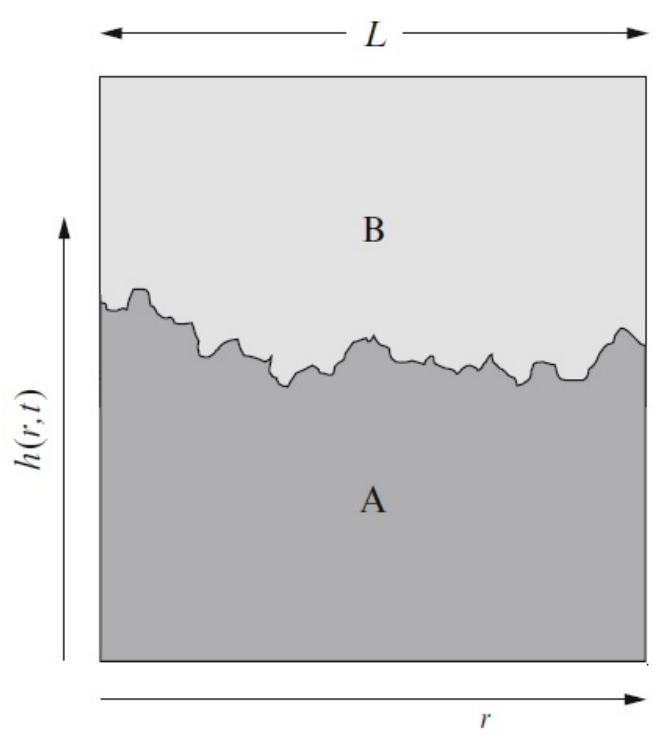

Figura 3.1: Formação de superfíce entre dois meios.[3] 
Para estudar analiticamente os modelos de crescimento muitos métodos têm sido desenvolvidos. Um em particular é bastante útil que consiste em associar uma equação de crescimento estócastico com um dado processo de crescimento. O objetivo é encontrar a variação temporal da altura da superfície $h(\mathbf{r}, t)$ em qualquer posição $\mathbf{r}$, onde $\mathbf{r}$ pertence a um substrato d-dimensional. Em geral, o crescimento pode ser descrito pela equação contínua

$$
\frac{\partial h(\mathbf{r}, t)}{\partial t}=\Phi(\mathbf{r}, t)
$$

onde o fluxo $\Phi(\mathbf{r}, t)$ é o número de partículas por unidade de tempo chegando na posição $\mathbf{r}$ da superfície no instante $t$.

O fluxo de partículas não é uniforme, uma vez que as partículas são depositadas em posições aleatórias. A aleatoriedade pode ser incorporada na teoria pela decomposição de $\Phi(\mathbf{r}, t)$ em dois termos de tal modo que a equação (3.1) torna-se

$$
\frac{\partial h(\mathbf{r}, t)}{\partial t}=F+\eta(\mathbf{r}, t)
$$

O primeiro termo, $F$, é o número médio de partículas chegando no sítio $\mathbf{r}$ e pode ser definido como um campo. O segundo termo, $\eta(\mathbf{r}, t)$, caracteriza as flutuações aleatórias no processo de deposição e é um número aleatório não correlacionado

$$
\left\langle\eta(\mathbf{r}, t) \eta\left(\mathbf{r}^{\prime}, t^{\prime}\right)=2 D \delta^{d}\left(\mathbf{r}-\mathbf{r}^{\prime}\right) \delta\left(t-t^{\prime}\right)\right.
$$

Onde $D$ é a dispersão e $d$ é a dimensão e possui valor médio nulo.

$$
\langle\eta(\mathbf{r}, t)\rangle=0
$$

Considere uma superfície caracterizada pela altura $h(\mathbf{r}, t)$ na figura (3.1), e assuma que não possua saliências na superfície em $t=0$. O objetivo é partindo da equação (3.2) derivar uma equação de crescimento para superfícies correlacionadas. Espera-se que esta equação de crescimento possua a forma 


$$
\frac{\partial h(\mathbf{r}, t)}{\partial t}=G(h, \mathbf{r}, t)+\eta(\mathbf{r}, t) .
$$

Onde $G(h, \mathbf{r}, t)$ é uma função geral que depende da altura da superfície, da posição, do tempo. Iremos estudar o comportamento da superfície representado na figura (3.1). Como nosso primeiro passo é obter a equação de crescimento vamos apresentar as simetrias básicas do problema.

\subsection{Príncipios de simetria}

\subsubsection{Invariância sob translação temporal}

A equação (3.5) descreve a forma geral da evolução da superfície onde $G$ corresponde a parte determinística e $\eta$ a parte estocástica. A equação de crescimento não deve depender da origem do tempo, de modo que a equação deve ser invariante sobre a transformação $t \rightarrow t+t_{0}$ não permitindo deste modo qualquer dependência explícita no tempo. Temos que a derivada $\partial h / \partial t$ é invariante sobre uma translação temporal, isto é, $\partial h / \partial\left(t+t_{0}\right)=\partial h / \partial t$.

\subsubsection{Invariância na translação ao longo da direção do crescimento}

A equação deve considerar o fato que o crescimento é independente da origem escolhida para localizar a posição da superfície ao longo da direção da altura $h$, então a equação de crescimento deve ser invariante sobre a transformação $h \rightarrow$ $h+h_{0}$. Portanto, $G$ não depende explicitamente de $h$ e a equação deve ser construída com combinações de $\nabla h, \nabla^{2} h, \ldots, \nabla^{n} h$. Nota-se que $\nabla\left(h+h_{0}\right)=\nabla h$, então o termo $\nabla h$ sobrevive nesta transformação. 


\subsubsection{Invariância na translação na direção perpendicular à direção do} crescimento

O crescimento é independente da origem escolhida para localizar $r$ ao longo da direção paralela à interface. A equação deve ser invariante com respeito a transformação $r \rightarrow r+r_{0}$. Desta forma, G não deve depender explicitamente de $r$ e pode conter somente combinações das seguintes derivadas:

$$
\frac{\partial}{\partial r_{x}}, \frac{\partial^{2}}{\partial r_{x}^{2}}, \frac{\partial^{3}}{\partial r_{x}^{3}}, \ldots, \frac{\partial^{n}}{\partial r_{x}^{n}}
$$

\subsubsection{Rotação e inversão na direção do crescimento}

Esta regra exclui todas as derivadas ímpares, isto é, excluindo vetores como $\nabla h, \nabla\left(\nabla^{2} h\right)$, etc. O termo $\nabla h$ sobrevive ao considerarmos os princípios anteriores, contudo não sobrevive a este último. Ao realizar a transformação $\mathbf{r} \rightarrow-\mathbf{r}$ teremos que $\partial h / \partial(-x)=-\partial h / \partial x$, i.e., a derivada troca de sinal sobre esta transformação, diferente dos termos com derivadas pares $(\nabla h)^{2}$ e $\nabla^{2} h$.

\subsubsection{Simetria Up/Down para a altura}

As flutuações nas superfícies são similares com respeito a altura média da superfície.Deste modo ao fazer a transformação $h \rightarrow-h$ excluí-se as potências pares de $h$, termos como $(\nabla h)^{2},(\nabla h)^{4}$, etc. Esta simetria está intimamente conectada com o equilíbrio natural da superfície e para problemas fora do equilíbrio esta simetria pode ser quebrada.

\subsection{A equação de Edwards-Wilkinson}

Para encontrar a forma da equação de crescimento devemos considerar todos os termos que podem ser formados pelas combinações de $\nabla^{n} h$. Um por um elimina- 
mos todos os termos que violam os princípios de simetrias tratados anteriormente. Portanto nós temos

$$
\frac{\partial h(\mathbf{r}, t)}{\partial t}=a_{1} \nabla^{2} h+a_{2} \nabla^{4} h+\ldots+a_{n} \nabla^{2 n} h+\eta(\mathbf{r}, t)
$$

O objetivo deste estudo está nas propriedades de escala focando no comportamento assintótico das funções que caracteriza a superfície $(t \longrightarrow \infty)$ e $(L \longrightarrow \infty)$. Observe que decompondo $h(\mathbf{r}, t)$ em série de Fourier

$$
h(\mathbf{r}, t)=\int_{-\infty}^{\infty} \frac{d \tau}{2 \pi} \int \frac{d^{d} \mathbf{k}}{(2 \pi)^{d}} h(\mathbf{k}, t) \exp ^{i(\mathbf{k} \cdot \mathbf{r}-\tau t)}
$$

$\nabla^{n} h(\mathbf{r}, t)$ corresponde a termos $k^{n} h(\mathbf{k}, \tau)$, logo no limite dos grandes comprimentos de onda $(\mathbf{k} \rightarrow 0)$, os termos dominantes serão os de menores expoentes em $\mathbf{k}$. Logo precisamos preservar apenas os termos de baixa ordem. Isto significa que somente estes termos influenciam nos valores dos expoentes que caracterizam o crescimento.

O termo de ruído, $\eta(\mathbf{r}, t)$ na equação (3.6) incorpora o caráter estocástico do processo de flutuação. Para um caso simples onde assumimos que não tenha correlação e que as propriedades do ruído sejam satisfeitas a equação mais simples que descreve as flutuações de uma interface em equilíbrio, chamada de equação de Edwards-Wilkinson (EW) ([17]) possui a forma:

$$
\frac{\partial h(\mathbf{r}, t)}{\partial t}=\nu \nabla^{2} h+\eta(\mathbf{r}, t)
$$

As principais características desta equação são as seguintes:

- Ela conserva a altura média $\bar{h}$.

- Quando o fluxo F é uniforme e constante, a equação não afeta as propriedades assintóticas da rugosidade. A equação de primeira ordem (3.7) contêm toda a Física do modelo de crescimento. Ela é suficiente para representar a classe de universalidade do modelo em estudo. 
- Ela possui a mesma forma da equação de difusão se omitirmos o termo de ruído. Do mesmo jeito que a equação de difusão a equação EW gradualmente elimina as irregularidades nas superfícies.

- O coeficiente $\nu$ desempenha o papel de uma tensão superficial. Quanto maior o valor de $\nu$ mais rápido será a suavização do sistema.

- Ela descreve corretamente o crescimento aleatório com relaxação, desde que o gradiente $\nabla h$ da superfície permaneça pequeno. Isto significa que:

$$
\delta h \ll \delta r .
$$

- Sabemos que $|\delta h| \sim|\delta r|^{\alpha}$ a partir da definição de $\alpha$. Para grandes distâncias esta condição portanto restringe $\alpha<1$.

\subsection{A equação de Kardar-Parisi-Zhang}

A equação de EW discutida anteriormente foi a primeira equação contínua usada para estudar o crescimento de superfícies por deposição de partículas. As previsões desta teoria linear alteram quando adicionamos termos não lineares na equação de crescimento. A primeira extensão da equação Edwards-Wilkinson para incluir termos não lineares foi proposto por Kardar, Parisi e Zhang (KPZ) ([18]).

Embora a equação KPZ não possa ser resolvida de forma fechada devido a sua característica não linear e estocástica, alguns poucos resultados exatos tem sido encontrados. Além disso, poderosos métodos de aproximação podem ser usados para obter mais conhecimento sobre as propriedades de escala e dos expoentes.

\subsubsection{Construção da equação KPZ}

Embora não se possa derivar formalmente a equação KPZ pode-se desenvolver um conjunto de argumentos plausíveis usando princípios físicos que motiva a 
adição de termos não lineares na teoria linear (3.7) e os princípios de simetria usados no caso da equação de EW.

O objetivo é construir uma equação contínua que descreva os modelos de crescimento, para isto iremos generalizar a equação de EW. Para exemplificar esta generalização poderemos tomar o modelo de deposição balística onde as partículas que chegam a superfície acabam grudando na primeira partícula que encontram ocasionando um crescimento lateral. Para incluir este crescimento lateral na equação de crescimento nós adicionamos uma nova partícula à superficie. O crescimento ocorre localmente normal à superfície gerando um incremento $\delta h$ ao longo do eixo $h$ que é decomposto em duas componentes: $v \delta t$ paralelo ao normal local da superfície e $|\nabla h| v \delta t$ perpendicular ao normal local - como visto na figura (3.2). Pelo teorema de Pitagoras extraímos uma expressão para $\delta h$

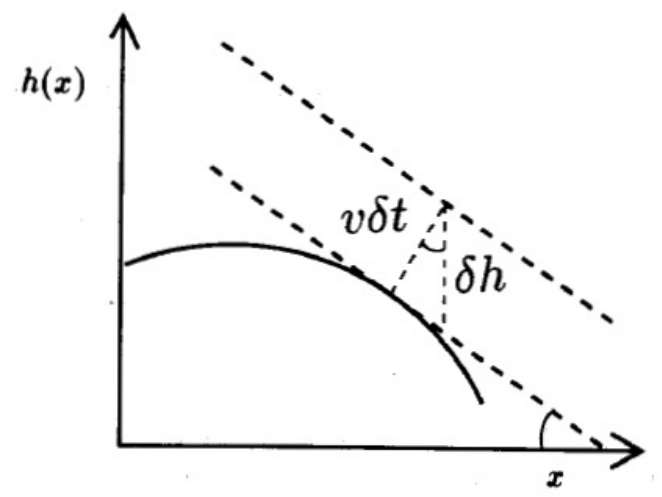

Figura 3.2: A origem do termo não linear na equação KPZ.

$$
\delta h=\left[(v \delta t)^{2}+(v \delta t \nabla h)^{2}\right]^{1 / 2}=v \delta t\left[1+(\nabla h)^{2}\right]^{1 / 2} .
$$

Se $|\nabla h| \ll 1$ podemos expandir a equação (3.8)

$$
\frac{\partial h(\mathbf{r}, t)}{\partial t}=v+\frac{v}{2}(\nabla h)^{2}+\ldots
$$

sugerindo que um termo não-linear na forma $(\nabla h)^{2}$ deve ser incluído na equação de crescimento para destacar o crescimento lateral. Adicionando este termo à equação 
EW obtemos a equação KPZ:

$$
\frac{\partial h(\mathbf{r}, t)}{\partial t}=\nu \nabla^{2} h+\frac{\lambda}{2}(\nabla h)^{2}+\eta(\mathbf{r}, t) .
$$

O primeiro termo do lado direito da (3.10) descreve a relaxação da interface através da tensão superficial $\nu$. O segundo termo é o termo não linear de menor ordem que pode aparecer na equação de crescimento.

\subsection{Propriedades da equação KPZ}

\subsubsection{Invariância de Galileu}

Esperamos que a equação KPZ (3.10) e a equação EW (3.7) sejam invariantes sobre as seguintes mudanças de escala: $\mathbf{r} \rightarrow b \mathbf{r}, h \rightarrow b^{\alpha} h$ e $t \rightarrow b^{z} t$, onde $b$ é o parâmetro de escala. Realizando estas transformações na equação KPZ vem

$$
\frac{\partial h(\mathbf{r}, t)}{\partial t}=\nu b^{z-2} \nabla^{2} h+\frac{\lambda}{2} b^{\alpha+z-2}(\nabla h)^{2}+b^{-\alpha+\frac{z-d}{2}} \eta(\mathbf{r}, t) .
$$

Para garantir a invariância esperamos que a equação (3.11) deva ser independente de $b$. Contudo, este procedimento nos fornece três relações, incompativéis entre si. Como o termo não linear é determinando nós desprezamos o termo $\nu \nabla^{2} h$. Assim obtemos as relações para os expoentes:

$$
\alpha=\frac{2-d}{3}, \quad \beta=\frac{2-d}{4+d},
$$

que não estão corretos, pois para $d=1$ eles fornecem $\alpha=1 / 3$ e $\beta=1 / 5$. Qualquer outra combinação das 3 equações nos leva a expoentes errados.

Então, onde foi que erramos? A razão é que, reescalonando nosso sistema os diferentes termos $(\nu, \lambda$ e $D)$ na equação de crescimento não são renormalizados independentemente - sendo relacionados um com o outro. Portanto, para ter uma escala invariante não devemos simplesmente assumir que os expoentes de $b$ são nulos 
na equação (3.11), uma vez que $\nu, D$ e $\lambda$ podem ser alterados durante o reescalonamento. Para melhor análise a partir da abordagem de grupo de renormalização apresentado em [2,3], onde após um longo processo é obtido as equações de fluxos dos parâmetros para KPZ, i.e.

$$
\begin{gathered}
\frac{d \nu}{d l}=\nu\left[z-2+K_{d} g^{2} \frac{2-d}{4 d}\right], \\
\frac{d D}{d l}=D\left[z-d-2 \alpha+K_{d} \frac{g^{2}}{4}\right], \\
\frac{d \lambda}{d l}=\lambda[\alpha+z-2] .
\end{gathered}
$$

Onde a constante de acoplamento é definida como

$$
g^{2} \equiv \frac{\lambda^{2} D}{\nu}
$$

A equação para o fluxo da constante de acoplamento é

$$
\frac{d g}{d l}=\frac{2-d}{2} g+K_{d} \frac{2 d-3}{4 d} g^{3}
$$

enquanto $K_{d} \equiv S_{d} /(2 \pi)^{d}$ e $S_{d}$ é a área da esfera d-dimensional. Os expoentes podem ser obtidos pelos pontos fixos não nulos das equações de fluxos, i.e.

$$
\frac{d \nu}{d l}=\frac{d D}{d l}=\frac{d \lambda}{d l}=0
$$

A partir da equação (3.14) obtemos a relação:

$$
\alpha+z=2
$$

Esta equação fornece uma relação entre o expoente de rugosidade $\alpha$ e o expoente dinâmico z que caracteriza o crescimento e é chamada de invariância de Galileu (IG).

A partir das equações (3.12-3.14), (3.15) e (3.16) obtemos as expressões para os expoentes: 


$$
\alpha=\frac{(2-d)^{2}}{6-4 d} \quad \beta=\frac{(2-d)^{2}}{2(6-4 d)-(2-d)^{2}}
$$

Para $d=1$ obtemos $\alpha=1 / 2$ e $\beta=1 / 3$ como era esperado. Para $d \geq 2$ os resultados não tem sentido físico. Para $d=2, \alpha=\beta=0$ e para $d>2$ eles são negativos. Deste modo a renormalização funciona apenas para $d=1$.

\subsection{Problemas atuais da Física de crescimento}

Pelo desenvolvimento anterior torna-se claro que a equação KPZ tornouse o melhor ponto de partida para o estudo de crescimento. Verifica-se também que nenhuma solução analítica foi apresentada por Kardar, Parisi e Zhang para sua equação. Além disso, a renormalização apresentada só vale para $d=1$, falhando desastrosamente para $d>1$. A equação KPZ vem sendo estudada nos últimos 30 anos por físicos e matemáticos, assim descrevemos abaixo alguns problemas atuais relativos a KPZ e modelos de crescimento.

\subsubsection{Validade da invariância de Galileu}

A primeira pergunta que aparece naturalmente é se a IG é válida para qualquer dimensão. Observe que na renormalização, a variação de $\lambda$, equação (3.14), aparece independente da dimensão $d$ e do acoplamento $g$, portanto a equação (3.18) é um forte candidato para ser um resultado exato.

Para dimensões fracionárias diversos autores mostraram[19, 20, 21] que a relação é violada para o modelo RSOS e de corrosão. Para dimensões inteiras resultados númericos recentes para o modelo de corrosão $[22,23]$ sugerem que a relação é válida. Entretando, até o momento não existe prova definitiva de sua validade para $d+1$ dimensões. 


\subsubsection{Dimensão crítica superior}

Foi questionado anteriormente se a invariância de Galileu era independente da dimensão do sistema, agora perguntamos se existe uma dimensão crítica superior para KPZ tal como para o modelo de Ising.

O modelo de Ising 1-D possui solução bem conhecida [24, 10], para o modelo em 2-D temos a conhecida solução de Onsager [25] e várias soluções numéricas para Ising 3-D [26, 27, 28]. Para $d=4$ a solução exata é igual a campo médio. Para $d>4$ os expoentes não mudam mais, sendo os mesmos de $d=4$. Neste caso dizemos que $d_{c}=4$ é a dimensão crítica superior (DCS) do modelo de Ising.

Nos últimos anos tem sido especulado [29, 30, 31, 32] que a DCS para o modelo KPZ é a mesma do modelo de Ising i.e. $d_{c}=4$. Rodrigues et al [22] mostraram através de métodos númericos que se $d_{c}$ existe, ela deve ser maior que 6 , i.e., $d_{c}>6$. Este resultado contribui eliminando a hipótese $d_{c}=4$, mas não resolve a questão fundamental se realmente existe uma DCS para KPZ.

\subsubsection{Autômatos celulares e KPZ}

É extremamente importante saber se os modelos de autômatos celulares proposta para KPZ realmente imitam KPZ. A condição mínima para isto é que pertençam a mesma classe de universalidade KPZ. Recentemente [1, 6] foi demonstrando que o modelo de autômato celular para corrosão pertence a classe de universalidade KPZ. Neste trabalho nosso foco está no estudo da teoria para modelos de autômatos celulares, especificamente o modelo RSOS, e assim desenvolver uma metodologia para obter os expoentes críticos. Por fim verificar que o modelo RSOS pertence a classe de universalidade KPZ. 


\section{Capítulo 4}

\section{Evolução da rugosidade em modelos de autômatos celulares}

Desde os trabalhos originais de Von Neumann no final dos anos 40 os modelos de autômatos celulares (MAC) têm sido amplamente usados [33, 34]. Um MAC é um modelo cujas as variáveis possuem valores discretos. No MAC a deposição e o movimento das partículas em sua superfíe obedecem a regras probabilísticas simples. Estas interações geram fenômenos coletivos semelhantes aos que estamos habituados em sistemas condensados. Consequentemente, surgem vários fenômenos em escala microscópica e macroscópica. Devido a sua simplicidade, eles têm encontrado aplicações em muitos campos como fluídos dinâmicos [35, 36], transição de fase [37] e jogo da vida [38].

Neste capítulo, apresentamos nossos principais resultados. Focaremos nos MAC para crescimento, principalmente no RSOS [5, 39] e iremos propor uma teoria para entender a evolução da rugosidade via MAC, onde obteremos seu comportamento para grandes sistemas $(L \rightarrow \infty)$. Além disso, tentaremos obter o expoente de rugosidade $\alpha$. 


\subsection{Modelo RSOS}

O modelo RSOS, introduzido por Kim e Kosterlitz, permitiu a primeira investigação sistemática dos expoentes de crescimento para altas dimensões [5]. O algoritmo de crescimento de Kim e Kosterlitz seleciona aleatoriamente um sítio em uma superfície d-dimensional, e aumenta-se a altura da superfície por um, $h_{i} \rightarrow$ $h_{i}+1$, desde que em cada etapa do crescimento a condição $|\Delta h|=0,1$ seja satisfeita entre o sítio selecionado e seus vizinhos.

Kim e Kosterlitz estudaram númericamente este modelo para $d \leq 4$. Eles se concentraram na determinação do expoente $\beta$, porque este expoente descreve o comportamento inicial da rugosidade e não exige a saturação do sistema (que para grande sistemas é muito demorado).

Existem varias formulações do modelo RSOS, nós usaremos o modelo RSOS representado na figura (4.1), onde o algoritmo é dado por

$$
\xi_{i}(t+\Delta t)=\max \left(\xi_{i-1}(t)+k, \xi_{i}(t)+1, \xi_{i+1}(t)+k\right)
$$

com $k=0$ para uma deposição lado a lado - parte b da figura (4.1) - e com $k=1$ para uma deposição diagonal - parte c da figura (4.1).

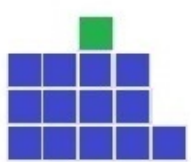

$i$

a

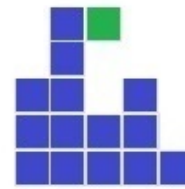

$i$

b

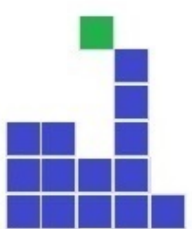

$i$

c

Figura 4.1: Configurações para o modelo RSOS.

Na figura (4.1) ocorre o seguinte. No caso a a altura do sítio $i$ escolhido é igual a de seus vizinhos, logo a partícula depositada adere a este sítio. No caso b e c a altura dos vizinhos do sítio $i$ é maior, logo a partícula depositada adere no 
substrato como mostra na figura ocasionando regiões com buracos após sucessivas deposições.

\subsubsection{Simulações do Modelo RSOS}

Antes de iniciarmos o estudo analítico do modelo RSOS foram efetuadas algumas simulações simples para o modelo RSOS. Usando o algoritmo (4.1), com $k=0$, repetimos as regras de $t=0$, até a saturação. Fazendo um média sobre diversos experimentos obtemos a rugosidade em função do tempo.

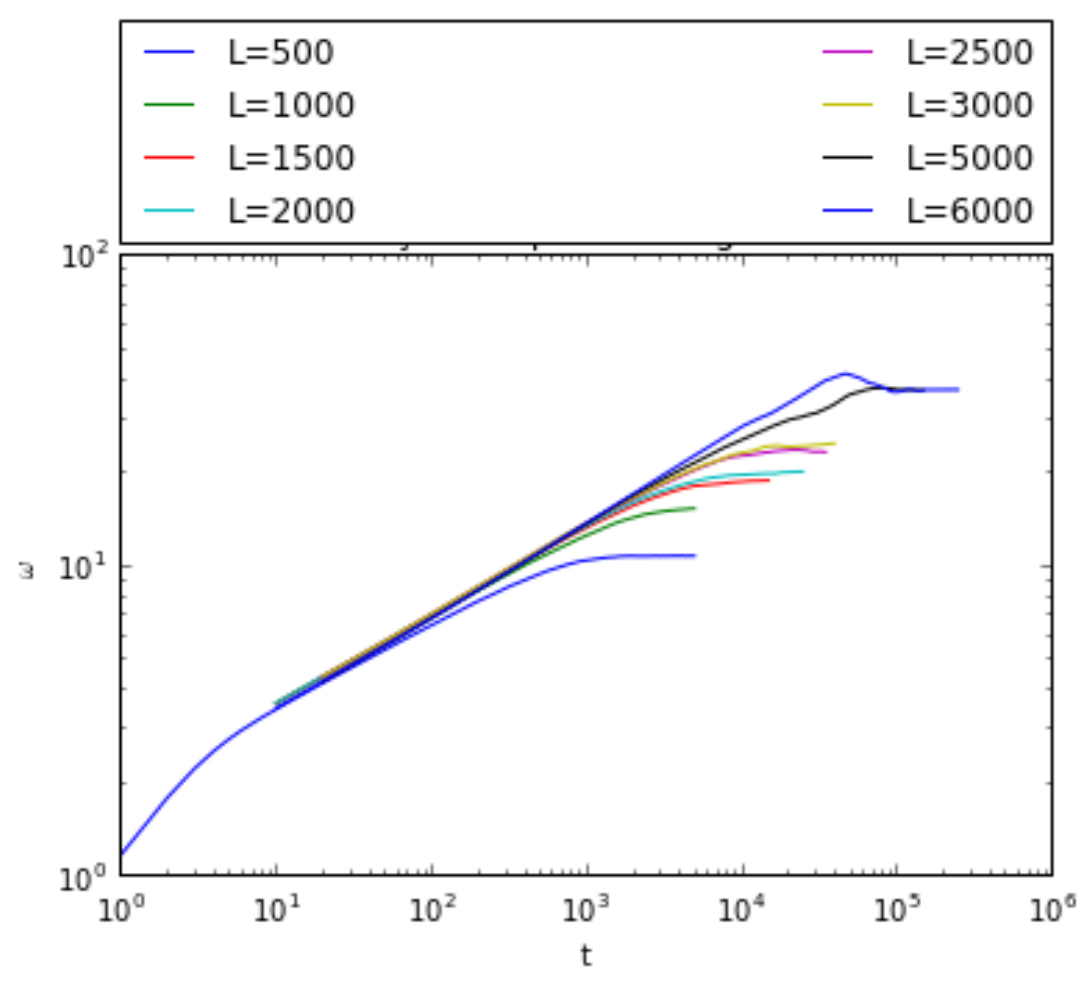

FigurA 4.2: Evolução temporal da rugosidade. 
Na figura (4.2) mostramos a evolução da rugosidade em função do tempo, para diversos comprimentos $L$ em escala logaritmica. Utilizamos as regras (a) e (b) da figura (4.1) e cada curva representa uma média sobre 20000 simulações distintas. Podemos observar no gráfico (4.2) que para cada valor de $L$ a saturação ocorre em tempos diferentes.

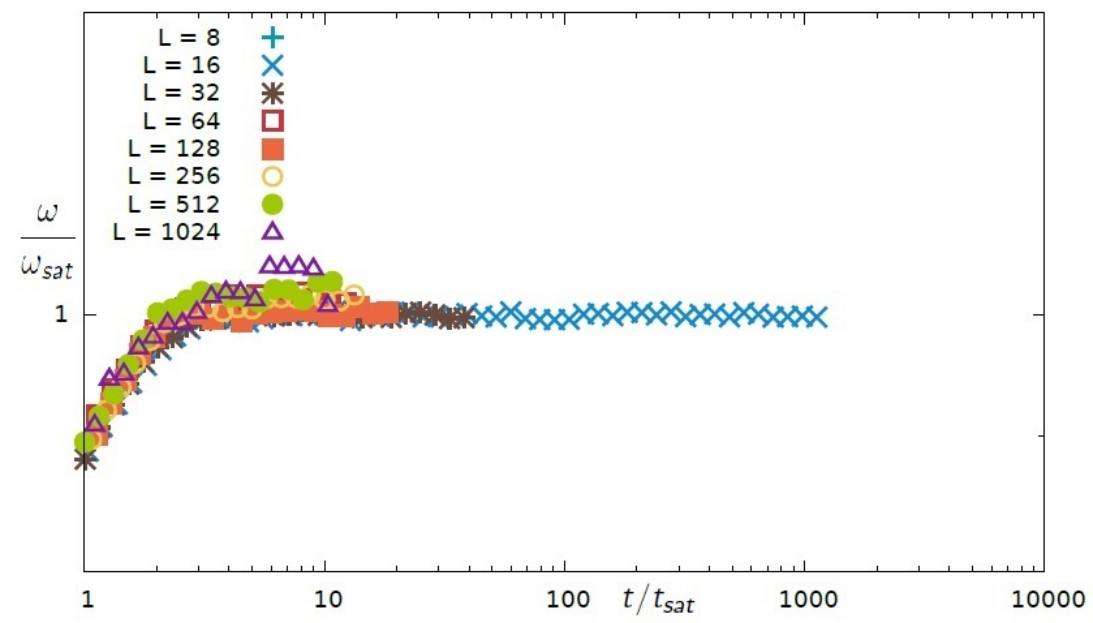

Figura 4.3: Evolução temporal da rugosidade (normalizado). O gráfico é uma gentiliza do Dr. Evandro A. Rodrigues

Na figura (4.3) temos a rugosidade normalizada $\omega(t) / \omega_{\text {sat }}$ em função do tempo normalizado $t / t_{\text {sat }}$. Na figura podemos observar o colapso dos dados, i.e., $\omega(t) / \omega_{\text {sat }}=f\left(t / t_{\text {sat }}\right)$ como descrito pela relação de Family-Vicsek (2.7). 


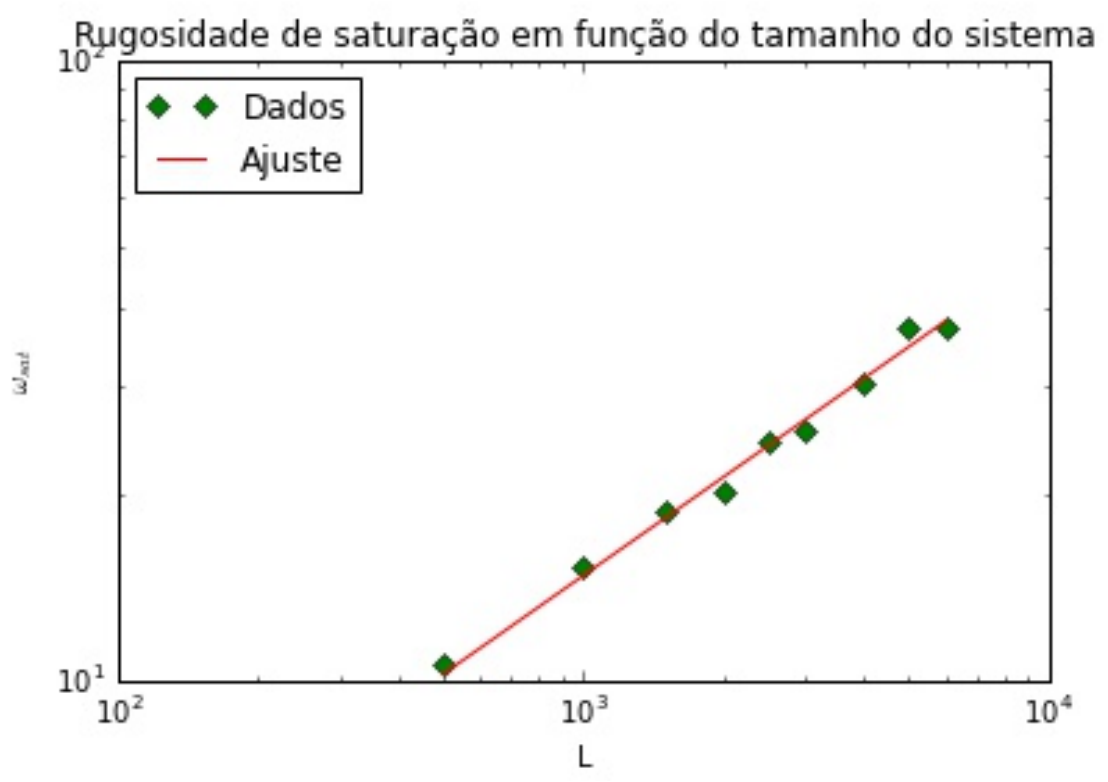

FigurA 4.4: $\omega_{\text {sat }}$ em função do comprimento $L$

Na figura (4.4) exibimos $\omega_{\text {sat }}$ em função do comprimento $L$. O ajuste dos pontos da função $\omega_{\text {sat }} \sim L^{\alpha}$ resulta em $\alpha=0.527 \pm 0.029$, i.e. próximo ao valor esperado de $\alpha=1 / 2$.

\subsection{Descrição da teoria para MAC}

Começamos definindo os conceitos gerais para o MAC. Primeiramente, em um instante $t$ escolhemos aleatoriamente um sítio $i$. Este procedimento fornece o caráter estocástico ao MAC. Então no instante seguinte $t+\Delta t$ definimos uma regra para alterar a altura do sítio $i$ ou de seus vizinhos $i \pm 1$. Então fixamos nossa atenção para o procedimento para obter a variação da rugosidade dada pela equação (2.2). Considerando uma séries de experimentos $j=1, \ldots, N$, a variação da rugosidade quadrática para um único passo do MAC atuando no sítio $i$ é representada por $\Delta \omega_{j}^{2}=\Delta \omega_{j}^{2}\left(\xi_{i-1}, \xi_{i}, \xi_{i+1}, t\right)$. Consequentemente a média de todas as configurações 
possíveis no limite $(N, L) \rightarrow \infty$ será

$$
\frac{d \omega^{2}(t)}{d t}=\lim _{\Delta t \rightarrow 0}\left\langle\frac{\Delta \omega^{2}}{\Delta t}\right\rangle=L \int f\left(\xi_{i-1}, \xi_{i} \xi_{i+1}, \omega\right) \Delta \omega^{2} \mathrm{~d} \xi_{i-1} \mathrm{~d} \xi_{i} \mathrm{~d} \xi_{i+1}
$$

onde $\Delta t=1 / L$ é o intervalo de tempo onde um processo ocorre. De fato, a média de todas as variações, para todos os experimentos e eventos possíveis é equivalente a fazer uma média sobre um ensemble no instante $t$. Mas determinar a Função de Distribuição de Probabilidade (FDP) de configurações $f$ não é uma tarefa fácil, Sasamoto e Spohn [40] resolveram a equação KPZ para uma dimensão. Eles encontraram que a FDP $g(h)$ da altura $h(\mathbf{r}, t)$ é dada pela distribuição Tracy-Widom [41].

Para nossa FDP não existe uma solução até o momento, e o motivo é simples: primeiro a FDP depende do MAC e da dimensão $d+1$, então não deve ser universal, segundo é uma FDP de configurações e não de alturas. Contudo há duas situações onde $f$ pode ser facilmente determinada. A situação mais simples é para tempo muito pequeno $t<1$ onde não há correlações e o processo é um simples caso de movimento browniano, por este motivo a FDP é uma função de distribuição equiprobabilidade (FDE) $f_{\text {eqp }}\left(\xi_{i-1}, \xi_{i}, \xi_{i+1}\right)$. Outra situação importante é quando $t \rightarrow \infty$, pois quando ocorre a saturação do sistema $\omega \rightarrow \omega_{\text {sat }}$ a derivada na equação (4.2) é nula. Uma vez que neste limite não há mudança na FDP, que nos levará para uma uma situação de equilíbrio. Para este caso novamente temos FDE.

\subsubsection{Obtenção da FDE}

Para obter a FDE nós devemos fazer algumas considerações: Primeiro, vamos definir

$$
\xi_{i}=h_{i}-\bar{h}
$$

como uma variável contínua. Esta hipótese é razoável, já que $\bar{h}$ é um número real, refazemos a média sobre vários experimentos, onde em cada um deles existe uma probabilidade de $h_{i}$ assumir um dado valor, e finalmente queremos considerar 
experimentos para $L$ grande onde as variações discretas tornam-se insignificante. Assim consideramoas também o conjunto de variáveis reais $\left(\xi_{1}, \xi_{2}, \ldots, \xi_{L}\right)$ como uma hiperesfera $S_{L}$ de dimensão $L$. Neste espaço uma hiperesfera de raio $\rho$ tem volume

$$
\Omega(\rho, L)=\frac{2 \pi^{(L+1) / 2}}{\Gamma[(L+1) / 2]} \rho^{L}
$$

onde $\Gamma$ é a função Gama. A equação (2.2) rescrita como

$$
\sum_{i=1}^{L} \xi_{i}^{2}=\omega^{2} L
$$

define a superfície da hiperesfera de dimensão $L-1$ e raio $R=\omega \sqrt{L}$ e $\xi_{i}(t)$ representa uma variável de valor médio nulo, isto é

$$
\sum_{i=1}^{L} \xi_{i}(t)=0
$$

que é a equação de um plano na hiperesfera $S_{L}$. A evolução dessas superfícies no hiper-espaço, representa a evolução da rugosidade $w(t)$. As restrições dadas pelas equações (2.2) e (4.6) reduzem a hiperesfera $S_{L}$ para uma hiperesfera $S_{L-2}$, que é o espaço de todas as possibilidades. Agora fixado $\left(\xi_{i-1}, \xi_{i}, \xi_{i+1}\right)$ define-se um subespaço $S_{L-5}$ de dimensão $L-5$ onde para esta hiperesfera a equação (2.2) torna-se

$$
\sum_{k=4}^{L} \xi_{k}^{2}(t)-\xi_{1}^{2}(t)-\xi_{2}^{2}(t)-\xi_{3}^{2}(t)=R^{2}-r^{2}=(R \cos (\psi))^{2}
$$

onde $r=R \sin (\psi)$. O $R \cos (\psi)$ é o raio da hiperesfera no hiperespaço $S_{L-5}$ e $\psi$ é um ângulo auxiliar, $0<\psi<\pi / 2$. Por conveniência podemos introduzir a mudança de coordenadas

$$
\left\{\begin{array}{l}
\xi_{i-1}=r \sin \theta \cos \varphi \\
\xi_{i}=r \sin \theta \sin \varphi \\
\xi_{i+1}=r \cos \theta
\end{array}\right.
$$

Como será demonstrado com detalhes no apêndice, a FDE de uma dada configuração é dada por

$$
f_{\text {eqp }}=\frac{\Omega(L-5, R \cos \psi)}{\Omega(L, R)}
$$


$\log 0$

$f_{\text {eqp }}\left(\omega, \xi_{i-1}, \xi_{i}, \xi_{i+1}\right) d \xi_{i-1} d \xi_{i} d \xi_{i+1}=\frac{\Gamma[(L-1) / 2]}{\pi^{3 / 2} \Gamma[(L-4) / 2]} \cos (\psi)^{L-4} \sin ^{2}(\psi) \sin (\theta) d \psi d \theta d \varphi$

Note que a equação (4.10) não depende de $\omega$, esta independência ocorre para qualquer dimensão $d+1$, onde um hipercubo tem $2^{d}$ vizinhos e gera um subespaço de $2^{d}+1$ variáveis. Logo a dependência $f_{\text {eqp }} \propto \omega^{-\left(2^{d}+1\right)}$ é compensada pelo elemento de volume que é proporcional a $\omega^{2^{d}+1}$.

Então a partir das equações (2.2) e (4.6) podemos escrever

$$
\Delta \omega^{2}=-(\Delta \bar{h})^{2}+\frac{1}{L} \sum_{i}\left[2 \xi_{i} \Delta h_{i}+\left(\Delta h_{i}\right)^{2}\right]
$$

A equação (4.11) é uma fórmula geral (independente do MAC) para o incremento da rugosidade.

\subsubsection{Normalização da probabilidade}

Para nossa análise consideramos que a função de probabilidade FDE dada pela equação (4.10) é válida para o regime de saturação. Para obter o valor da rugosidade de saturação consideramos sistemas com tamanhos muito grande $L \rightarrow \infty$, isto se deve que nesta região a função de probabilidade é normalizada. Integrando a equação da $\operatorname{FDE}(4.10)$ em todo o espaço $(0<\psi<\pi / 2,0<\theta<\pi, 0<\varphi<2 \pi)$ :

$$
N(L)=\int_{0}^{\pi / 2} \int_{0}^{\pi} \int_{0}^{2 \pi} \frac{\Gamma[(L-1) / 2]}{\pi^{3 / 2} \Gamma[(L-4) / 2]} \cos (\psi)^{L-4} \sin ^{2}(\psi) \sin (\theta) d \psi d \theta d \varphi,
$$

obtemos

$$
N(L)=\frac{\Gamma[(L-1) / 2] \Gamma[(L-3) / 2]}{\Gamma[(L-4) / 2] \Gamma[L / 2]} .
$$

A normalização da probabilidade $N(L)$ é uma função do tamanho do sistema $L$. Observe que

$$
\lim _{L \rightarrow \infty} N(L)=1
$$




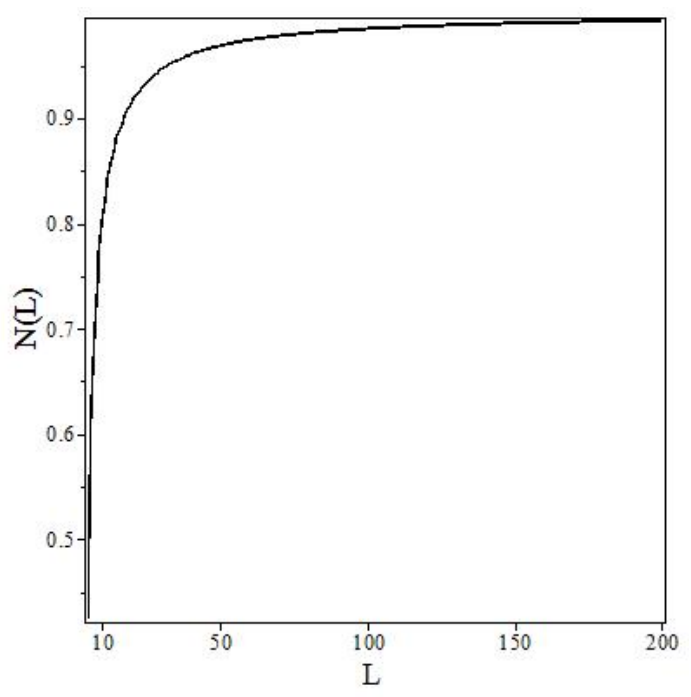

FigurA 4.5: Normalização da probabilidade em função de $L$

Na figura (4.5) plotamos $N(L)$ em função de $L$. Obseve que somente para grandes valores de $L$ a FDE será normalizada, e podemos falar em uma teoria contínua. Para valores pequenos de $L$ muitas vezes temos que fazer correções dos chamados efeitos de comprimentos finitos (Size finite effects [29]).

\subsubsection{Considerações gerais sobre a teoria}

Partindo da equação (4.11) podemos afirmar que próximo a saturação

$$
\frac{d \omega^{2}(t)}{d t}=P_{n}(\omega)
$$

onde $P_{n}(\omega)$ é um polinômio de grau $n=0,2$. Considerando a simetria da superfície (4.5) podemos esperar $n=2$, e que $P_{2}(-\omega)=P_{2}(\omega)$. Mais ainda, considerando que derivada acima é nula apenas para $\omega=\omega_{\text {sat }}$, podemos afirmar que $P_{2}(\omega)=$ $c\left(\omega_{\text {sat }}^{2}-\omega^{2}\right)$ onde $c>0$. Neste caso obtemos

$$
\omega(t)=\omega_{s} g\left(\frac{t}{t_{s a t}}\right)=\omega_{s} g\left(\frac{t}{t_{s a t}}\right)^{\beta}
$$

onde $g(x)=1-\exp (-x)$ e aqui $\beta=1 / 2$. Obviamente o valor de $\beta$ está errado, já que não esperamos que (4.16) seja válida para tempos intermediários onde a correlação 
está presente, i.e. a relação é válida para $t<1$ e para $t>t_{\text {sat }}$. Não sendo válida na região onde $\beta$ é definido $1<t<t_{\text {sat }}$. Entretanto, podemos usar (4.16) como uma relação empírica e ela ajusta bem os dados da simulação. Observe que ela satisfaz a relação de Family-Vicsek. Mais ainda, ela sugere uma relação mais geral que Family-Vicsek, i.e.

$$
g\left(t / t_{\text {sat }}\right)=\left[\omega(t) / \omega_{\text {sat }}\right]^{1 / \beta},
$$

é uma relação universal independente da dimensão. Isto foi recentemente observado por Rodrigues, Mello e Oliveira [22], para o modelo de corrosão em $d+1$ dimensões para $1 \leqslant d \leqslant 6$. A relação (4.17), embora não esteja totalmente comprovada, é uma das contribuições da nossa teoria.

\subsection{Aplicação da teoria para o modelo RSOS}

A partir da equação do incremento da rugosidade (4.11) iremos analisar a evolução temporal da rugosidade para o modelo RSOS representado na figura (4.1), e cuja evolução e dada pelo algoritmo (4.1). Desde modo escolhendo $i=2$ podemos apresentar todas as configurações possíveis para o modelo RSOS na tabela (4.1)

\begin{tabular}{|l|l|l|l|}
\hline Caso & Limites & $\Delta \xi_{2}$ & $L \Delta \omega^{2}$ \\
\hline a & $\xi_{2} \geq\left(\xi_{1}, \xi_{3}\right)$ & 1 & $2 \xi_{2}+\left(1-\frac{1}{L}\right)$ \\
\hline $\mathrm{b}$ & $\xi_{2 \pm 1}>\left(\xi_{2}, \xi_{2 \mp 1}\right)$ & $\xi_{2 \pm 1}-\xi_{2}$ & $\xi_{2 \pm 1}^{2}-\xi_{2}^{2}-\frac{1}{L}\left(\xi_{2 \pm 1}-\xi_{2}\right)^{2}$ \\
\hline $\mathrm{c}$ & $\xi_{2 \pm 1}>\left(\xi_{2}, \xi_{2 \mp 1}\right)$ & $\xi_{2 \pm 1}-\xi_{2}+1$ & $\xi_{2 \pm 1}^{2}-\xi_{2}^{2}+2 \xi_{2 \pm 1}-\frac{1}{L}\left[\left(\xi_{2 \pm 1}-\xi_{2}\right)^{2}+\right.$ \\
& & & $\left.+2\left(\xi_{2 \pm 1}-\xi_{2}\right)\right]+\left(1-\frac{1}{L}\right)$ \\
\hline
\end{tabular}

TABELA 4.1: Configurações possíveis para o modelo RSOS 
A partir de agora iremos analisar o incremento da rugosidade para os casos $a$ e $b$ da figura (4.1) onde $k=0$.

\subsubsection{Caso a}

Neste primeiro caso estamos considerando o limite $\xi_{2}>\left(\xi_{1}, \xi_{3}\right)$, então podemos escrever a equação da rugosidade usando as equações (4.2) e (4.11):

$$
\frac{d \omega^{2}(t)}{d t}=\int_{b_{1}}^{b_{2}} \int_{a_{1}}^{\xi_{2}} \int_{c_{1}}^{\xi_{2}}\left(2 \xi_{2}+(1-1 / L)\right) f\left(\xi_{1}, \xi_{2}, \xi_{3}, \omega\right) d \xi_{1} d \xi_{3} d \xi_{2}
$$

onde $a_{1}, \xi_{2}, c_{1}, \xi_{2}, b_{1}$ e $b_{2}$ são os limites de integração das variáveis $\xi_{1}, \xi_{3}$ e $\xi_{2}$, respectivamente.

Inserindo na equação (4.18) a equação (4.10) e fazendo a mudança de coordenadas definida na equação (4.8) teremos:

$$
\begin{aligned}
& \frac{d \omega^{2}(t)}{d t}=\pi^{-3 / 2} \frac{\Gamma[(L-1) / 2]}{\Gamma[(L-4) / 2]} \int_{0}^{\pi / 2} 2 R(\cos \psi)^{L-4}(\sin \psi)^{3} d \psi \iint(\sin \theta)^{2} \sin \varphi d \theta d \varphi+ \\
& \quad+\pi^{-3 / 2} \frac{\Gamma[(L-1) / 2]}{\Gamma[(L-4) / 2]}(1-1 / L) \int_{0}^{\pi / 2}(\cos \psi)^{L-4}(\sin \psi)^{2} d \psi \iint \sin \theta d \theta d \varphi
\end{aligned}
$$

onde já sabemos que $R=\sqrt{L} \omega$. Resolvendo a integral em $\psi$ na equação (4.19) temos:

$$
\frac{d \omega^{2}}{d t}=\pi^{-3 / 2} \frac{\Gamma[(L-1) / 2]}{\Gamma[(L-4) / 2]}\left\{\frac{2 \sqrt{L} A_{1}}{(L-3)(L-1)} \omega+\frac{\pi^{1 / 2}}{4} \frac{(1-1 / L) \Gamma[(L-3) / 2]}{\Gamma[L / 2]} A_{2}\right\}
$$

nesta equação $A_{1}$ e $A_{2}$ são os valores das integrais nos ângulos $\theta$ e $\varphi$ e não influenciam a dependência em $L$, podemos simplificar e escrever a equação (4.20) na forma

$$
\frac{d \omega^{2}(t)}{d t}=\lambda_{1} \omega+\lambda_{0}
$$

onde

$$
\begin{gathered}
\lambda_{1}=\pi^{-3 / 2} \frac{\Gamma[(L-1) / 2]}{\Gamma[(L-4) / 2]} \frac{2 \sqrt{L}}{(L-3)(L-1)} A_{1} \\
\lambda_{0}=\pi^{-3 / 2} \frac{\Gamma[(L-1) / 2]}{\Gamma[(L-4) / 2]} \frac{\pi^{1 / 2}}{4} \frac{(1-1 / L) \Gamma[(L-3) / 2]}{\Gamma[L / 2]} A_{2}
\end{gathered}
$$




\subsubsection{Caso b}

Devido a simetria entre os sítios $\xi_{1}, \xi_{2}$ e $\xi_{3}$, o que acontece de um lado acontece do outro, então podemos fazer somente a análise do caso b, i.e., quando $\xi_{1}>\left(\xi_{2}, \xi_{3}\right)$. Então a partir das equações (4.2) e (4.11)

$$
\frac{d \omega^{2}(t)}{d t}=\int_{a_{1}}^{a_{2}} \int_{b_{1}}^{\xi_{1}} \int_{c_{1}}^{\xi_{1}}\left(\xi_{1}^{2}-\xi_{2}^{2}-\frac{1}{L}\left(\xi_{1}-\xi_{2}\right)^{2}\right) f\left(\xi_{1}, \xi_{2}, \xi_{3}, \omega\right) d \xi_{2} d \xi_{3} d \xi_{1}
$$

Novamente faremos uso da simetria do problema. Ao calcular o valor médio do incremento da rugosidade temos o seguinte caso

$$
\left\langle\xi_{i}^{2}\right\rangle=\left\langle\xi_{i \pm 1}^{2}\right\rangle
$$

simplificando a equação (4.22). Inserindo na equação (4.22) a equação (4.10) e fazendo a mudança de coordenadas definida na equação (4.8) teremos

$\frac{d \omega^{2}}{d t}=-\pi^{-3 / 2} \frac{\Gamma[(L-1) / 2]}{\Gamma[(L-4) / 2]} \int_{0}^{\pi / 2} R^{2}(\cos \psi)^{L-4}(\sin \psi)^{4} d \psi \iint(\sin \theta)^{3}(\cos \varphi-\sin \varphi)^{2} d \theta d \varphi$

onde $R=\sqrt{L} \omega$. Resolvendo a integral em $\psi$ na equação (4.24)

$$
\frac{d \omega^{2}}{d t}=-\frac{3 \pi^{-3 / 2}}{8} \frac{\Gamma[(L-1 / 2)] \Gamma[(L-3) / 2]}{\Gamma[(L-4) / 2] \Gamma[(L / 2+1)]} B_{1} \omega^{2}
$$

onde $B_{1}$ são os valores das integrais nos ângulos $\theta$ e $\varphi$. Podemos escrever a equação (4.25) na forma

$$
\frac{d \omega^{2}}{d t}=-\lambda_{2} \omega^{2}
$$

onde

$$
\lambda_{2}=\frac{3 \pi^{-3 / 2}}{8} \frac{\Gamma[(L-1 / 2)] \Gamma[(L-3) / 2]}{\Gamma[(L-4) / 2] \Gamma[(L / 2+1)]} B_{1} .
$$

Por fim, adicionado as equações (4.21) e (4.26) podemos escrever a equação da variação da rugosidade total do sistema como

$$
\frac{d \omega^{2}}{d t}=-\lambda_{2} \omega^{2}+\lambda_{1} \omega+\lambda_{0}=P_{2}(\omega)
$$

O fato de $P_{2}(\omega)$ ser um polinômio de grau 2 é geral e independe do modelo de crescimento. Desta forma podemos calcular o valor do expoente de rugosidade $\alpha$. 


\subsection{Obtenção do expoente de rugosidade}

Para o cálculo da variação da rugosidade utilizamos a função probabilidade definida na equação (4.10) onde lidamos com dois casos: primeiro com sistemas sem correlação $t<1$ e segundo para grandes tempos $t \rightarrow \infty$ no qual ocorre a saturação da superfície. Para obter o valor de $\alpha$ vamos considerar o segundo caso.

Quando consideramos que ocorreu o processo de saturação observamos pelo gráfico da figura (2.2) que nesta região onde $t \gg t_{\text {sat }}$ a rugosidade não varia mais com o tempo, logo a equação da variação temporal da rugosidade dada pela equação (4.27) será nula, i.e. $P_{2}\left(\omega_{\text {sat }}\right)=0$, deste modo encontramos a seguinte solução

$$
\omega_{s a t}=\frac{\lambda_{1}}{2 \lambda_{2}}+\sqrt{\left(\frac{\lambda_{1}}{2 \lambda_{2}}\right)^{2}+\frac{\lambda_{0}}{\lambda_{2}}} .
$$

Então no contínuo $L \rightarrow \infty$ o valor da rugosidade de saturação é dado por $\omega_{\text {sat }} \propto L^{\alpha}$. Analisando os valores de $\lambda_{2}, \lambda_{1}$ e $\lambda_{0}$ e usando a fórmula de Stirling (ver Apêndice) temos:

$$
\begin{aligned}
\lambda_{2} & \rightarrow L^{-1} \\
\lambda_{1} & \rightarrow L^{0} \\
\lambda_{0} & \rightarrow L^{0}
\end{aligned}
$$

Substituindo estes limites na equação (4.28) temos então

$$
\omega_{\text {sat }} \propto L^{1}
$$

que difere do valor esperado de $\alpha=1 / 2$ para os modelos que pertencem a classe KPZ.

\subsubsection{O destino de uma teoria}

Uma teoria é tão boa quanto os resultados que ela prediz. O valor encontrado para o coeficiente de rugosidade $\alpha=1$ difere do valor para $\alpha$ dos modelos 
KPZ. Isto se deve ao fato que a distribuição que foi utilizada para resolver a equação (4.2) não possui simetria suficiente para que o valor de $\lambda_{1}$ seja nulo e desta forma pela equação (4.28) obteríamos $\omega_{\text {sat }} \propto L^{1 / 2}$. Deste modo chegamos a conclusão que a distribuição de equiprobabilidade não funciona para o caso RSOS, como funcionou para o modelo de corrosão $[1,7]$.

Por outro lado, a discusão geral que leva a equação (4.16) nos mostra que a teoria tem uma certa capacidade de predição, e sugere a existência de uma lei mais forte que a de Family-Vicsek. Existe ainda uma situação muito simples que pode ser resolvida pela teoria, trata-se da deposição balistica normal, que consiste no caso (a) da figura (4) sem a restrição dos primeiros vizinhos neste caso a integral resulta em

$$
\frac{d \omega^{2}}{d t}=P_{0}(\omega)=\text { Constante }
$$

$\log \omega(t) \propto t^{1 / 2}$ para todos os valores de $t$. Ou seja um movimento browniano simples. Observe que $P_{0}(\omega)$ não tem raiz i.e não apresenta saturação. Neste caso a nossa teoria reproduz o resultado correto.

Como vemos a teoria nos leva ao resultado correto em diversas situações, mas ainda não está completa a ponto de responder todas questões. No caso do modelo RSOS a questão resume-se a obter a distribuição de configurações correta. Iniciamos agora uma nova busca para uma função dinâmica que possa representar o modelo RSOS. Esta busca, entretanto, vai além dos objetivos dessa dissertação. 


\section{Capítulo 5}

\section{Conclusão}

Neste trabalho dedicamos nossa atenção ao estudo da dinâmica de crescimento na interface entre dois meios. Fizemos inicialmente uma revisão da teoria apresentando aos leitores interessados os conceitos básicos para analisar qualquer tipo de crescimento. Definimos os principais expoentes que aparece nos modelos de crescimeno, i.e. os expoentes críticos, $\alpha, \beta$ e $z$. Mostramos também que esses expoentes não são independentes, i.e existem leis de escala entre eles capítulo (2). Por exemplo $z=\frac{\alpha}{\beta}$. Deste modo, necessitamos conhecer apenas dois dos expoentes. No capítulo (3) tratamos do estudo da dinâmica de crescimento onde introduzimos as equações de crescimento EW e KPZ. A normalização da equação KPZ permitiu um novo desenvolvimento na Física, apresentando pela primeira vez uma solução exata para os expoentes crítícos em $1+1$ dimensões, i.e. $\alpha=1 / 2, \beta=1 / 3$ e $z=3 / 2$. A renormalização da equação KPZ nos leva também a famosa relação $\alpha+z=2$ conhecida como invariança de Galileu (IG). Chamamos a atenção para os problemas atuais no estudo de modelos de crescimento como a a validade da IG, se existe uma dimensão critica superior para KPZ, e a relação entre KPZ e autômatos celulares.

No capítulo (4), objetivo principal deste trabalho, apresentamos a teoria do autômato celular para o estudo da evolução de superfície, focando na evolução da rugosidade. Usando como base esta teoria focamos na analise do modelo RSOS onde 
obtemos o valor do expoente de rugosidade $\alpha$, contudo o resultado encontrado difere bastante do valor esperado de $\alpha=1 / 2$. Concluímos que a função de distribuição de configurações utilizada não é apropriada ao modelo. Este valor é mais apropriado a um variante do modelo RSOS[42]. Investigaremos se o resultado discutido aqui pode ser aplicado a este modelo.

Concluímos que a teoria do autômato celular é bastante útil para descrever a evolução temporal da rugosidade independentemente da regra que rege o modelo de crescimento, permitindo encontrar o valor dos expoente $\alpha$ e os demais utilizando as leis escala dada pela equação (2.9) e a IG. Para os trabalhos futuros temos o objetivo de encontrar uma função de probabilidade que nos permita encontrar corretamente os valores dos expoentes e desta forma analisar o modelo RSOS para dimensões $1+1$ e $2+1$. 


\section{Apêndice A}

\section{Incremento de rugosidade}

No capítulo (4) apresentamos a equação (4.11) que descreve a variação da rugosidade para um modelo de crescimento. A partir dos conceitos de altura média e rugosidade definidos no capítulo (2) demonstraremos a seguir os passos para obter a equação do incremento da rugosidade $[1,7]$.

\section{A.1 Desenvolvimento do incremento da rugosidade}

Suponha que uma partícula caia em um substrato de rugosidade $\omega$. Nosso principal objetivo é tentar prever qual será o incremento da rugosidade nesse substrato. Consideremos o substrato discreto descrito na figura (2.1), considerando o sistema antes de uma deposição e depois de uma deposição, a altura referente à posição $i$ de cada partícula, no referencial da altura média, será dada por

$$
\begin{aligned}
& \text { Antes: }: \omega^{2}(t)=\frac{1}{L} \sum_{i=1}^{L} \xi_{i}^{2}(t) \\
& \text { Depois: }: \omega^{2}(t+1)=\frac{1}{L} \sum_{i=1}^{L} \xi_{i}^{2}(t+1)
\end{aligned}
$$

onde $\xi_{i}(t)$ é definido na equação (2.3). Definiremos ainda:

$$
\Delta h_{i} \Rightarrow \text { o acréscimo da altura na posição } i
$$




$$
\Delta \bar{h} \Rightarrow \text { o acréscimo da altura média, }
$$

Então abrindo o termo do somatorio da segunda equação (A.1) temos

$$
\begin{array}{r}
\xi_{i}(t+1)^{2}=\left[h_{i}(t+1)-\bar{h}(t+1)\right]^{2} \\
\xi_{i}(t+1)^{2}=\left[h_{i}(t)+\Delta h_{i}-\bar{h}(t)-\Delta \bar{h}\right]^{2} \\
\xi_{i}(t+1)^{2}=\left[\xi_{i}(t)+\Delta h_{i}-\Delta \bar{h}\right]^{2} \\
\xi_{i}(t+1)^{2}=\xi_{i}(t)^{2}+\left[\Delta h_{i}-\bar{h}(t)^{2}\right]^{2}+2 \xi_{i}(t)\left[\Delta h_{i}-\bar{h}(t)\right] .
\end{array}
$$

Substituindo na equação (A.2) a primeira equação de (A.1) e definindo

$$
\begin{gathered}
\Delta \omega^{2}(t) \equiv \omega^{2}(t+1)-\omega^{2}(t) . \\
\Delta \omega^{2}(t)=\frac{1}{L} \sum_{i=1}^{L} \Delta \xi_{i}^{2},
\end{gathered}
$$

onde definimos $\Delta \xi_{i}(t)^{2}=\left[\Delta h_{i}-\bar{h}(t)^{2}\right]^{2}+2 \xi_{i}(t)\left[\Delta h_{i}-\bar{h}(t)\right]$. Onde reescrevendo temos

$$
\Delta \xi_{i}^{2}(t)=2 \xi(t) \Delta h_{i}(t)-2 \xi_{i}(t) \Delta \bar{h}-2 \Delta h_{i} \Delta \bar{h}+(\Delta \bar{h})^{2}+\left(\Delta h_{i}\right)^{2}
$$

Pela definição de $\xi_{i}$ na equação (4.3) temos que $\sum_{i} \xi_{i}=0$ e também $\sum_{i} \Delta h_{i}=L \Delta \bar{h}$. Considerando estas definições e usando a equação (A.4) temos

$$
\Delta \omega^{2}(t)=-(\Delta \bar{h})^{2}+\frac{1}{L} \sum_{i=1}^{L}\left[2 \xi_{i} \Delta h_{i}+\left(\Delta h_{i}\right)^{2}\right] .
$$

A equação (A.5) representa a fórmula geral para o incremento da rugosidade quadrática, independente do algoritmo do modelo. Para obter $\Delta \omega^{2}(t)$ é necessário conhecer os valores de $\Delta h_{i}$ e $\Delta \bar{h}$. 


\section{Apêndice B}

\section{Função de distribuição de equiprobabilidade (FDE)}

\section{B.1 Obtenção da função $f\left(\xi_{1}, \xi_{2}, \xi_{3}, \omega\right)$}

Para uma dada rugosidade, há um número infinito de configurações, determinadas pelas equações:

$$
\begin{gathered}
\xi_{1}^{2}+\xi_{2}^{2}+\ldots+\xi_{L}^{2}=L \omega^{2} \\
\xi_{1}+\xi_{2}+\ldots+\xi_{L}=0
\end{gathered}
$$

$\operatorname{logo}$ os valores de $\xi_{i}$, com $i=1,2, \ldots, L$ devem satisfazer às equações do sistema (B.1). Percebe-se que a primeira das equações refere-se à definição da rugosidade, enquanto que a segunda está associada à flutuação da altura.

Por analogia, as equações acima formam a chamada:

$$
\begin{array}{r}
\text { hiperesfera de dimensão } \mathrm{n}=L-1 \text { e } \\
\text { raio } \mathrm{R}=\left(L \omega^{2}\right)^{1 / 2} .
\end{array}
$$

Denotaremos essa hiperesfera de $H_{T}$, pois todas as configurações para uma dada rugosidade $\omega$ estão inseridas na superfície dessa hiperesfera, que corresponde a nossa hiperárea superficial ou hipersuperfície, cujá área total é denotada por $A_{T} \mathrm{e}$ cuja dimensão é dada por $n=L-2$. 
Para um dado $\xi_{1}, \xi_{2}$ e $\xi_{3}$ formamos uma outra hiperesfera, cuja superfície possui dimensão $n=L-5$. Esta hiperesfera é a figura obtida pela intersecção da equação (B.1) com os planos de $\xi_{1}, \xi_{2}$ e $\xi_{3}$. Sua área parcial $A_{p}$ é proporcional ao número de configurações que possue $\xi_{1}, \xi_{2}$ e $\xi_{3}$, isso significa que a àrea $A_{p}$ está conectada aos $\xi_{1}, \xi_{2}$ e $\xi_{3}$. Logo para cada $\xi_{1}, \xi_{2}, \xi_{3}$ e $\omega$, a área $A_{p}$ será diferente, significando um número diferente de configurações. Assim, a FDE de ocorrer uma dada configuração $\xi_{1}, \xi_{2}$ e $\xi_{3}$ será descrita por:

$$
f\left(\xi_{1}, \xi_{2}, \xi_{3} \omega\right) d \xi_{1} d \xi_{2} d \xi_{3}=\frac{A_{p}}{A_{T}} d \xi_{1} d \xi_{2} d \xi_{3}
$$

O cálculo da área total $A_{T}$ e da área parcial $A_{p}$ foi um trabalho elaborado por Washington Alves Soares et al apresentando em [1, 6]. Neste trabalhos têm-se a área total

$$
A_{T}=\frac{2 \pi^{\frac{L-1}{2}}}{\Gamma\left(\frac{L-1}{2}\right)}\left(L \omega^{2}\right)^{\frac{L-2}{2}},
$$

e a área parcial é

$$
A_{p}=\frac{2 \pi^{\frac{L-4}{2}}}{\Gamma\left(\frac{L-4}{2}\right)}\left[\left(L \omega^{2}-\left(\xi_{1}^{2}+\xi_{2}^{2}+\xi_{3}^{2}\right)\right)^{1 / 2}\right]^{L-5} .
$$

Logo inserindo as equações (B.3) e (B.4), na equação (B.2) obtem-se a FDE

$$
f\left(\xi_{1}, \xi_{2}, \xi_{3}, \omega\right)=\nu(L) \frac{\left(L \omega^{2}-\left(\xi_{1}^{2}+\xi_{2}^{2}+\xi_{3}^{2}\right)\right)^{\frac{L-5}{2}}}{\left(L \omega^{2}\right)^{\frac{L-2}{2}}},
$$

onde $\nu(L)=\pi^{-\frac{3}{2}} \frac{\Gamma\left(\frac{L-1}{2}\right)}{\Gamma\left(\frac{L-4}{2}\right)}$.

A FDE (B.5) pode ser escrita em coordenadas esféricas, como mostrado na equação (4.8):

$$
f(\omega, \psi, \theta, \varphi)=\nu(L)\left(L \omega^{2}\right)^{-\frac{3}{2}}(\cos \psi)^{L-5}
$$

E o elemento de volume da integral (4.2) em coordenadas esfericas, dadas pela equação (4.8), calculando o jacobiano $J_{\psi \theta \varphi}$ :

$$
d \xi_{1} d \xi_{2} d \xi_{3}=\left(L \omega^{2}\right)^{\frac{3}{2}}\left(\cos \psi \sin ^{2} \psi \sin \theta\right) d \psi d \theta d \varphi
$$


Concluíndo, a probabilidade propriamente dita como usamos no capítulo (4) em coordenadas esféricas:

$$
f\left(\omega, \xi_{1}, \xi_{2}, \xi_{3}\right) d \psi d \theta d \varphi=\nu(L)(\cos \psi)^{L-4} \sin ^{2} \psi \sin \theta d \psi d \theta d \varphi
$$




\section{Apêndice $\mathrm{C}$}

\section{Função Gama}

A função gama $\Gamma$ está presente em diversos ramos da Física. Durante este trabalho, precisamente do capítulo 4 fizemos bastante uso desta função matemática e de suas propriedades, principalmente da função beta, também chamada de integral de Euler de primeiro tipo, e da fórmula de Stirling. Neste apêndice iremos expor rapidamente sobre a função gamma e estas propriedades.

\section{C.1 Função Gama}

A Função Gama, denotada por $\Gamma(x)$, estende a idéia do fatorial a partir da faixa dos inteiros não-negativos e é definida pela integral imprópria

$$
\Gamma(x+1)=\int_{0}^{\infty} t^{x} e^{-t} d t .
$$

A integral é absolutamente convertegente para $x \geq 1$ uma vez que

$$
t^{x-1} e^{-t} \leq e^{-t / 2} \text { para } t \gg 1
$$

e $\int_{0}^{\infty} e^{-t / 2} d t$ é convergente.

Integrando por partes a integral (C.1) com $d v=e^{-t} d t$ e $u=t^{x}$ obtemos a integral funcional

$$
\Gamma(x+1)=x \Gamma(x) \operatorname{para} x>0 .
$$


Podemos facilmente calcular

$$
\left\{\begin{array}{l}
\Gamma(1)=\int_{0}^{\infty} e^{-t}=1 \\
\Gamma(2)=1 \cdot \Gamma(1)=1 \\
\Gamma(3)=3 \cdot \Gamma(2)=2 ! \\
\vdots
\end{array}\right.
$$

e finalmente,

$$
\Gamma(n+1)=n !
$$

Neste trabalho expressamos os coeficientes $\left(\lambda_{0}, \lambda_{1}, \lambda_{2}\right)$ do polinômio (4.27) em termos de funções gama, onde o argumento destas funções é o tamanho $L$ do substrato. No contínuo fazemos o limite $L \rightarrow \infty$ e neste caso o cálculo de (C.1) se torna muito trabalhoso, então usamos uma simples aproximação, chamada fórmula de Stirling que iremos demonstrar a seguir.

\section{C.1.1 Fórmula de Stirling}

Considerando o integrando $F \equiv t^{x} e^{-t}$ quando $x$ é muito grande. Então $t^{x}$ é uma função crescente de $t$, enquanto $e^{-t}$ é uma função decrescente de $t$. Logo o produto $F \equiv t^{x} e^{-t}$ apresenta um pico máximo em algum ponto $t=t_{0}$ e cai rapidamente quando $t$ se afasta de $t_{0}$. Podemos encontrar este ponto de máximo calculando a derivada do logaritmo de $F$.

$$
\begin{aligned}
\frac{d \ln F}{d t} & =0 \\
\frac{d}{d t}(x \ln t-t)=\frac{x}{t}-1 & =0 \\
t_{0} & =x
\end{aligned}
$$

Mas somente os valores de $t$ na vizinhança de $t_{0}=x$ contribui para o valor da integral (C.1). Então podemos analisar a função $F \equiv t^{x} e^{-t}$ expandindo-a próximo 
de $t=x+\delta$, onde $\delta \ll x$.

$$
\log F=x \ln t-t=x \ln (x+\delta)-(x+\delta)
$$

Expandindo o logaritmo em séries de Taylor,

$$
\ln (x+\delta)=\ln x+\ln \left(1+\frac{\delta}{x}\right)=\ln x+\frac{\delta}{x}-\frac{1}{2} \frac{\delta^{2}}{x^{2}}+\cdots
$$

Substituindo a equação (C.6) na (C.5) temos

$$
\begin{array}{r}
\ln F=x \ln x-x-\frac{1}{2} \frac{\delta^{2}}{x} \\
F=x^{x} e^{-x} e^{-\delta^{2} / 2 x} .
\end{array}
$$

A última exponencial mostra que $F$ possui um máximo em $\delta=0$ e torna-se muito pequena quando $|\delta| \gg \sqrt{x}$. Pela equação (C.7) a integral (C.1) torna-se

$$
\Gamma(x+1)=\int_{-x}^{\infty} x^{x} e^{-x} e^{-\delta^{2} / x} \delta=x^{x} e^{-x} \int_{-\infty}^{\infty} e^{-\delta^{2} / x} d \delta=\sqrt{2 \pi x} x^{x} e^{-x} \operatorname{para} x \gg 1
$$

Nesta última integral trocamos o limite inferior $-x$ por $-\infty$, devido que na região $\delta<-x$ o integrando é bem pequeno. Onde usamos a identidade

$$
\int_{-\infty}^{\infty} e^{-\alpha u^{2}} d u=\sqrt{\frac{\pi}{\alpha}}
$$

\section{C.1.2 Função Beta}

No capítulo (4) aos estudarmos os casos referentes as configurações apresentadas na tabela (4.1). As integrais nas equações (4.19) e (4.24) na variavel $\psi$ são as conhecidas funções betas que iremos demonstrar a seguir.

Da definição da função gama (C.1) podemos escrever:

$$
\Gamma(z) \Gamma(\xi)=\int_{0}^{\infty} e^{-t} t^{z-1} d t \int_{0}^{\infty} e^{-u} u^{\xi-1} d u,
$$


usando a seguinte mudança de variáveis: $t=x^{2} \rightarrow d t=2 x d x$ e $u=y^{2} \rightarrow d u=2 y d y$ e substituindo na expressão acima, encontramos

$$
\begin{array}{r}
\Gamma(z) \Gamma(\xi)=\int_{0}^{\infty} e^{-x^{2}}\left(x^{2}\right)^{z-1} 2 x d x \int_{0}^{\infty} e^{-y^{2}}\left(y^{2}\right)^{\xi-1} 2 y d y= \\
\Gamma(z) \Gamma(\xi)=4\left(\int_{0}^{\infty} \int_{0}^{\infty} e^{-\left(x^{2}+y^{2}\right)} x^{2 z-1} y^{2 \xi-1} d y d x\right) .
\end{array}
$$

Agora é conveniente introduzirmos as coordenadas polares $x=\rho \cos \theta$ e $y=\rho \sin \theta$ e o jacobiano $d x d y=\rho d \rho d \theta$ teremos

$$
\Gamma(z) \Gamma(\xi)=4\left(\int_{0}^{\infty} e^{-\rho^{2}} \rho^{2(z+\xi) d \rho} \int_{0}^{\frac{\pi}{2}}(\cos \theta)^{2 z-1}(\sin \theta)^{2 \xi-1} d \theta\right) .
$$

É fácil ver que

$$
\Gamma(z+\xi)=2 \int_{0}^{\infty} e^{-\rho^{2}} \rho^{2(z+\xi)-1} d \rho .
$$

Usando a equação (C.11) na equação (C.10) teremos que

$$
\Gamma(z) \Gamma(\xi)=\Gamma(z+\xi) \int_{0}^{\frac{\pi}{2}}(\cos \theta)^{2 z-1}(\sin \theta)^{2 \xi-1} d \theta,
$$

onde definimos a função beta:

$$
\beta(m, n)=2 \int_{0}^{\frac{\pi}{2}}(\cos \theta)^{2 m-1}(\sin \theta)^{2 n-1} d \theta .
$$

Finalmente demonstramos que

$$
\beta(z, \xi)=\frac{\Gamma(z) \Gamma(\xi)}{\Gamma(z+\xi)}
$$

Concluindo assim nossa demonstração. 


\section{Referências Bibliográficas}

[1] W. S. Alves, B. A. Mello, H. A. Fernandes, F. A. Oliveira, and I. V. L. Costa. Exact solution for the etching model. to be published, 2016. (document), 1, 2.2, 3.5.3, 4.4.1, A, B.1

[2] A. L. Barabási and H. E. Stanley. Fractal concepts in surface growth. Cambridge university press, 1995. (document), 2, 2.2, 2.3, 2.2, 3.4.1

[3] A. Lesne and M. Laguës. Scale invariance: From phase transitions to turbulence. Springer Science \& Business Media, 2011. (document), 3.1, 3.4.1

[4] L. B. da Silva Volk, N. P. Cogo, and E. V. Streck. Erosão hídrica influenciada por condições físicas de superfície e subsuperfície do solo resultantes do seu manejo, na ausência de cobertura vegetal. Bras. Ci. Solo, 28:763-774, 2004. 1, 3

[5] J. M. Kim and J. M. Kosterlitz. Growth in a restricted solid-on-solid model. Phys. Rev. Lett., 62:2289-2292, May 1989. 1, 4, 4.1

[6] Washington Soares Alves. Metodologia analítica para o modelo de corrosão: crescimento e rugosidade. Dissertação de Mestrado, Pós Graduaçáo em Ciências dos materiais - Universidade de Brasília, Brasília-DF, 2014. 1, 3.5.3, B.1 
[7] B. A. Mello, A. S. Chaves, and F. A. Oliveira. Discrete atomistic model to simulate etching of a crystalline solid. Phy. Rev. E, 63(4):041113, 2001. 1, 3, 4.4.1, A

[8] R. L. Puurunen. Random deposition as a growth mode in atomic layer deposition. C. V. D., 10(3):159-170, 2004. 2

[9] Z-W Lai and S. Das Sarma. Kinetic growth with surface relaxation: Continuum versus atomistic models. Phy. Rev. Lett., 66(18):2348, 1991. 2

[10] S. R. Salinas. Introdução a Física Estatística Vol. 09. Edusp, 1997. 2.1.2, 3.5.2

[11] F. Family and T. Vicsek. Scaling of the active zone in the Eden process on percolation networks and the ballistic deposition model. J. Phy. A. : Mathematical and General, 18(2):L75, 1985. 2.2

[12] Ismael Victor de Lucena Costa. Processos estocásticos: difusão e crescimento. Tese de Doutorado, Instituto de Física - Universidade de Brasilia, Brasilia-DF, 2006. 3

[13] Evandro Alves Rodrigues. Correlação de velocidade em um modelo de corrosão. Dissertação de Mestrado, Instituto de Física - Universidade de Brasília, Brasília-DF, 2008. 3

[14] N. Provatas, T. Ala-Nissila, M. Grant, K. R. Elder, and L. Piché. Flame propagation in random media. Phy. Rev. E, 51(5):4232, 1995. 3

[15] André Telles Campos. Propagação de fogo e equações estocásticas. Tese de Doutorado - Instituto de Física - Universidade de Brasília, Brasília-DF, 2013. 3

[16] André Telles Campos. Modelamento da propagação superficial de frentes de fogo com a equação KPZ. Dissertação de Mestrado, Instituto de Física - Universidade de Brasília, Brasília-DF, 2009. 3 
[17] S. F. Edwards and DR. Wilkinson. The surface statistics of a granular aggregate. In Proceedings of the Royal Society of London A: Mathematical, Physical and Engineering Sciences, volume 381, pages 17-31. The Royal Society, 1982. 3.2

[18] M. Kardar, G. Parisi, and Y. C. Zhang. Dynamic scaling of growing interfaces. Phy. Rev. Lett., 56(9):889, 1986. 3.3

[19] Z. Xun, G. Tang, L. Song, K. Han, H. Xia, D. Hao, and Y. Yang. Dynamic scaling behaviors of the Etching model on fractal substrates. Journal of Statistical Mechanics: Theory and Experiment, 2014(12):P12008, 2014. 3.5.1

[20] S. B. Lee, H. C. Jeong, and J. M. Kim. A restricted solid-on-solid model for growth on fractal substrates. Journal of Statistical Mechanics: Theory and Experiment, 2008(12):P12013, 2008. 3.5.1

[21] Z. Xun, H. Xia, L. Wu, L. Song, Z. Zhang, D. Hao, and G. Tang. A fractal Langevin equation describing the kinetic roughening growth on fractal lattices. Journal of Statistical Mechanics: Theory and Experiment, 2015(8):P08016, 2015. 3.5.1

[22] E. A. Rodrigues, F. A. Oliveira, and B. A. Mello. On the Existence of an Upper Critical Dimension for Systems Within the KPZ Universality Class. Acta Physica Polonica B, 46:1231, 2015. 3.5.1, 3.5.2, 4.2.3

[23] E. A. Rodrigues, B. A. Mello, and F. A. Oliveira. Growth exponents of the etching model in high dimensions. J. Phy. A: Mathematical and Theoretical, 48(3):035001, 2015. 3.5.1

[24] K. Huang. Statistical mechanics. 1963. 3.5.2

[25] L. Onsager. Crystal statistics. I. A two-dimensional model with an orderdisorder transition. Phy. Rev., 65(3-4):117, 1944. 3.5.2 
[26] A. L. Talapov and H. W. J. Blöte. The magnetization of the 3D Ising model. J. Phy. A: Mathematical and General, 29(17):5727, 1996. 3.5.2

[27] R. Guida and J. Zinn-Justin. 3d Ising model: the scaling equation of state. Nuclear Physics B, 489(3):626-652, 1997. 3.5.2

[28] S. El-Showk, M. F. Paulos, D. Poland, S. Rychkov, D. Simmons-Duffin, and A. Vichi. Solving the 3D Ising model with the conformal bootstrap. Phy. Rev. D, 86(2):025022, 2012. 3.5.2

[29] Evandro Alves Rodrigues. Dinâmica de corrosão: expoentes críticos, invariância de Galileu e dimensão superior. Tese de Doutorado, Instituto de Física - Universidade de Brasília, Brasília-DF, 2014. 3.5.2, 4.2.2

[30] E. Marinari, A. Pagnani, G. Parisi, and Z. Rácz. Width distributions and the upper critical dimension of Kardar-Parisi-Zhang interfaces. Phy. Rev. E, 65(2):026136, 2002. 3.5.2

[31] F. Colaiori and M. A. Moore. Upper critical dimension, dynamic exponent, and scaling functions in the mode-coupling theory for the Kardar-Parisi-Zhang equation. Phy. Rev. Lett., 86(18):3946, 2001. 3.5.2

[32] M. Schwartz and E. Perlsman. Upper critical dimension of the Kardar-ParisiZhang equation. Phy. Rev. E, 85(5):050103, 2012. 3.5.2

[33] S. et al Wolfram. Theory and applications of cellular automata, volume 1. World scientific Singapore, 1986. 4

[34] J. Kari. Theory of cellular automata: A survey. Theoretical computer science, 334(1):3-33, 2005. 4

[35] S. Chen and G. D. Doolen. Lattice Boltzmann method for fluid flows. Annual review of fluid mechanics, 30(1):329-364, 1998. 4 
[36] R. Benzi, S. Succi, and M. Vergassola. The lattice Boltzmann equation: theory and applications. Phy. Rep., 222(3):145-197, 1992. 4

[37] H. Hinrichsen. Non-equilibrium critical phenomena and phase transitions into absorbing states. Advances in physics, 49(7):815-958, 2000. 4

[38] G. Szabó and G. Fath. Evolutionary games on graphs. Phy. Rep., 446(4):97216, 2007. 4

[39] A. Pagnani and G. Parisi. Multisurface coding simulations of the restricted solid-on-solid model in four dimensions. Phy. Rev. E, 87(1):010102, 2013. 4

[40] T. Sasamoto and H. Spohn. One-Dimensional Kardar-Parisi-Zhang Equation: An Exact Solution and its Universality. Phys. Rev. Lett., 104:602, February 2010. 4.2

[41] C. A. Tracy and H. Widom. Level-spacing distributions and the airy kernel. Communications in Mathematical Physics, 159(1):151-174, 1994. 4.2

[42] R. G. da Silva, M. L. Lyra, C. R. da Silva, and G. M. Viswanathan. Roughness scaling and sensitivity to initial conditions in a symmetric restricted ballistic deposition model. The European Physical Journal B-Condensed Matter and Complex Systems, 17(4):693-697, 2000. 5 